\title{
Deep wide-field near-infrared survey of the Carina Nebula ${ }^{\star}$
}

\author{
T. Preibisch ${ }^{1}$, T. Ratzka ${ }^{1}$, B. Kuderna ${ }^{1}$, H. Ohlendorf ${ }^{1}$, R. R. King ${ }^{2}$, S. Hodgkin ${ }^{3}$, \\ M. Irwin ${ }^{3}$, J. R. Lewis ${ }^{3}$, M. J. McCaughrean ${ }^{2,4}$, and H. Zinnecker ${ }^{5,6,7}$
}

\author{
${ }^{1}$ Universitäts-Sternwarte München, Ludwig-Maximilians-Universität, Scheinerstr. 1, 81679 München, Germany \\ e-mail: preibisch@usm.uni-muenchen.de \\ 2 Astrophysics Group, College of Engineering, Mathematics and Physical Sciences, University of Exeter, Exeter EX4 4QL, UK \\ 3 Cambridge Astronomical Survey Unit, Institute of Astronomy, Madingley Road, Cambridge, CB3 0HA, UK \\ 4 European Space Agency, Research \& Scientific Support Department, ESTEC, Postbus 299, 2200 AG Noordwijk, The Netherlands \\ 5 Astrophysikalisches Institut Potsdam, An der Sternwarte 16, 14482 Potsdam, Germany \\ ${ }^{6}$ Deutsches SOFIA Institut, Universität Stuttgart, Pfaffenwaldring 31, 70569 Stuttgart, Germany \\ 7 NASA-Ames Research Center, MS 211-3, Moffett Field, CA 94035, USA
}

Received 25 February 2011 / Accepted 4 April 2011

\section{ABSTRACT}

\begin{abstract}
Context. The Great Nebula in Carina is a giant $\mathrm{H}$ II region and a superb location in which to study the physics of violent massive star formation, but the population of the young low-mass stars remained very poorly studied until recently.

Aims. Our aim was to produce a near-infrared survey that is deep enough to detect the full low-mass stellar population (i.e. down to $\approx 0.1 M_{\odot}$ and for extinctions up to $A_{\mathrm{V}} \approx 15 \mathrm{mag}$ ) and wide enough to cover all important parts of the Carina Nebula complex (CNC), including the clusters $\operatorname{Tr} 14,15$, and 16 as well as the South Pillars region.

Methods. We used HAWK-I at the ESO VLT to survey the central $\approx 0.36 \mathrm{deg}^{2}$ area of the Carina Nebula. These data reveal more than 600000 individual infrared sources down to magnitudes as faint as $J \approx 23, H \approx 22$, and $K_{\mathrm{s}} \approx 21$. The results of a recent deep X-ray survey (which is complete down to stellar masses of $\sim 0.5-1 M_{\odot}$ ) are used to distinguish between young stars in Carina and background contaminants. We analyze color-magnitude diagrams (CMDs) to derive information about the ages and masses of the low-mass stars.

Results. The ages of the low-mass stars agree with previous age estimates for the massive stars. The CMD suggests that $\approx 3200$ of the X-ray selected stars have masses of $M_{*} \geq 1 M_{\odot}$; this number is in good agreement with extrapolations of the field IMF based on the number of high-mass $\left(M_{*} \geq 20 M_{\odot}\right)$ stars and shows that there is no deficit of low-mass stars in the CNC. The HAWK-I images confirm that about $50 \%$ of all young stars in Carina are in a widely distributed, non-clustered spatial configuration. Narrowband images reveal six molecular hydrogen emission objects (MHOs) that trace jets from embedded protostars. However, none of the optical $\mathrm{HH}$ objects shows molecular hydrogen emission, suggesting that the jet-driving protostars are located very close to the edges of the globules in which they are embedded.

Conclusions. The near-infrared excess fractions for the stellar population in Carina are lower than typical for other, less massive clusters of similar age, suggesting that the process of circumstellar disk dispersal proceeds on a faster timescale in the CNC than in the more quiescent regions, most likely due to the very high level of massive star feedback in the CNC. The location of all but one of the known jet-driving protostars at the edges of the globules adds strong support to the scenario that their formation was triggered by the advancing ionization fronts.
\end{abstract}

Key words. stars: formation - stars: luminosity function, mass function - circumstellar matter - stars: pre-main sequence ISM: individual objects: NGC 3372 - ISM: jets and outflows

\section{Introduction}

Most stars in the Galaxy are thought to be born in massive star-forming regions (e.g., Blaauw 1964; Miller \& Scalo 1978; Briceño et al. 2007), and therefore in close proximity to massive stars. This also applies to the origin of our solar system, for which recent investigations found convincing evidence that it formed in a large cluster, consisting of (at least) several thousand stars, and that the original solar nebula was directly affected by nearby massive stars (e.g., Adams 2010). As a consequence, the role of environment is now an essential topic in studies of star and planet formation. The presence of high-mass stars can lead to physical conditions that are vastly different from those in regions where only low-mass stars form. The very

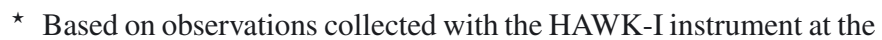
VLT at Paranal Observatory, Chile, under ESO program 60.A-9284(K). luminous O-type stars profoundly influence their environment by their strong ionizing radiation, powerful stellar winds, and, finally, by supernova explosions. This feedback can disperse the surrounding natal molecular clouds (e.g., Freyer et al. 2003), and thus terminate the star formation process (=negative feedback). However, ionization fronts and expanding superbubbles can also compress nearby clouds and thereby trigger the formation of new generations of stars (=positive feedback; e.g., Gritschneder et al. 2010; Preibisch \& Zinnecker 2007). These processes determine the key outputs from star formation, including the stellar mass function, the total star formation efficiency, as well as the evolution of circumstellar disks around the young stars (e.g., Clarke 2007) and the frequency of planetary formation. While this general picture is now well established, the details of the feedback processes, i.e. cloud dispersal on the one hand, and triggering of star formation on the other hand, are still only poorly understood. A fundamental problem is that nearly 
all massive star-forming clusters with high levels of feedback are quite far away and therefore difficult to study. At distances greater than $5 \mathrm{kpc}$, the detection and characterization of the full stellar populations is very difficult (if not impossible); often, only the bright high- and intermediate-mass stars can be studied, leaving the low-mass $\left(M \leq 1 M_{\odot}\right)$ stars unexplored or even undetected. However, since the low-mass stars constitute the vast majority of the stellar population, a good knowledge of the lowmass stellar content is essential for any inferences on the initial mass function and for understanding the nature of the star formation process. Detailed information about the low-mass stars and their protoplanetary disks is also crucial for investigations of the interaction between the high- and low-mass stars. The intense UV radiation from massive stars may remove considerable amounts of the circumstellar material from nearby young stellar objects (YSOs, hereafter). This may limit the final masses of the low-mass stars (see Whitworth \& Zinnecker 2004) and should also affect the formation of planets (see, e.g., Throop \& Bally 2005).

In this context, the Great Nebula in Carina (NGC 3372, see, e.g., Smith \& Brooks 2008, for an overview) provides a unique target for studies of massive star feedback. At a very well known and moderate distance of $2.3 \mathrm{kpc}$, the Carina Nebula Complex (CNC, hereafter) represents the nearest southern region with a large massive stellar population (65 O-type stars, see Smith 2006). Among these are several of the most massive $\left(M \gtrsim 100 M_{\odot}\right)$ and luminous stars known in our Galaxy, e.g., the famous Luminous Blue Variable $\eta$ Car, the O2 If* star HD 93129Aa, several O3 main sequence stars and Wolf-Rayet stars. The CNC is the most nearby region that samples the top of the stellar mass function. The presence of stars with $M \gtrsim 100 M_{\odot}$ implies that the level of feedback in the CNC is already close to that in more extreme extragalactic starburst regions, while at the same time its comparatively moderate distance guarantees that we still can study details of the cluster and cloud structure at good enough spatial resolution and detect and characterize the low-mass stellar populations. Due to this unique combination of properties, the CNC represents the best galactic analog of giant extragalactic $\mathrm{H}$ II and starburst regions.

Most of the very massive stars in the CNC reside in several loose clusters, including $\operatorname{Tr} 14,15$, and 16, for which ages between $\sim 2$ and $\sim 8 \mathrm{Myr}$ have been estimated (see Smith \& Brooks 2008). The CNC is thus often denoted as a "cluster of clusters". In the central region, the molecular clouds have already been largely dispersed by the feedback from the numerous massive stars. Southeast of $\eta$ Car, in the so-called "South Pillars" region, the clouds are eroded and shaped by the radiation and winds from $\eta$ Car and $\operatorname{Tr} 16$, giving rise to numerous giant dust pillars, which feature very prominently in the mid-infrared images made with the Spitzer Space Observatory (Smith et al. 2010b). The complex contains more than $10^{5} M_{\odot}$ of gas and dust (see Yonekura et al. 2005; Preibisch et al. 2011a), and deep infrared observations show clear evidence of ongoing star formation in these clouds. Several deeply embedded YSOs (e.g., Mottram et al. 2007) and a spectacular young cluster (the "Treasure Chest Cluster", see Smith et al. 2005) have been found. A deep $H S T \mathrm{H} \alpha$ imaging survey revealed dozens of jet-driving YSOs (Smith et al. 2010a), and Spitzer surveys located numerous embedded intermediate-mass protostars throughout the Carina complex (Smith et al. 2010b; Povich et al. 2011a). The formation of this substantial population of very young ( $\leq 1 \mathrm{Myr}$ ), partly embedded stars was probably triggered by the advancing ionization fronts that originate from the (several Myr old) high-mass stars. The CNC provides an excellent target to investigate these effects on the formation and evolution of low-mass stars (and their forming planetary systems) during the first few Myr.

While the un-obscured population of high-mass stars $(M \geq$ $20 M_{\odot}$ ) in the CNC is well known and characterized, the (much fainter) low-mass $\left(M \leq 1 M_{\odot}\right)$ stellar population remained largely unexplored until now. One reason for this is the faintness of the low-mass stars: at a distance of $2.3 \mathrm{kpc}$, an age of $3 \mathrm{Myr}$, and assuming typical extinctions of $A_{\mathrm{V}}=3.5 \mathrm{mag}$ (Preibisch et al. 2011b), stars with masses of $0.5 M_{\odot}\left[0.1 M_{\odot}\right]$ are predicted to have magnitudes of $V=23.6$ [26.6], $J=17.0$ [19.2], and $K=15.5$ [17.7] (see Baraffe et al. 1998). The existing nearinfrared (NIR) observations were either too shallow to detect the full low-mass population (e.g., Sanchawala et al. 2007), or covered only very small parts of the complex (e.g. Ascenso et al. 2007).

This was the motivation for the deep wide-field NIR survey presented in this paper. Our survey is $\geq 5$ mag deeper than the 2MASS data, deep enough to detect essentially all young stars in the CNC, and can be used to derive information about the ages and masses of the young low-mass stars. A fundamental restriction is that the NIR data alone do not allow us to distinguish young stars in the $\mathrm{CNC}$ from the numerous galactic field stars in the area (see discussion in Sect. 2). For this purpose, we use the recent results from the Chandra Carina Complex Project (CCCP), that has mapped the entire extent of the CNC $\left(\approx 1.4 \mathrm{deg}^{2}\right)$ in X-rays. A complete overview of the CCCP can be found in Townsley et al. (2011) and associated papers in a Special Issue of the Astrophysical Journal Supplements. The CCCP data led to the detection of 14368 individual X-ray sources. Based on a statistical analysis of the properties of each individual source, Broos et al. (2011b) classified 10714 of these as young stars in the CNC. The identification of HAWK-I infrared counterparts of the X-ray sources in the CNC and a few basic aspects of the X-ray selected population of young stars have been recently presented in Preibisch et al. (2011b). In this paper we discuss the complete set of HAWK-I data (Sect. 2) and present a detailed analysis of the NIR color-magnitude diagrams (Sect. 3). In Sect. 4 we analyze the NIR excess fractions for the different clusters in the CNC. In Sect. 5 we draw global implications on the size of the stellar population and the IMF of the CNC. In Sect. 6 we look at the spatial distribution of the young stars and list newly discovered stellar clusters in the CNC. Section 7 contains the results of our search for protostellar jets in $\mathrm{H}_{2}$ narrow-band images. Global conclusions on the star formation process in the CNC are discussed in Sect. 8.

\section{HAWK-I observations and data analysis}

The NIR imager HAWK-I (see Kissler-Patig et al. 2008) at the ESO $8 \mathrm{~m}$ Very Large Telescope is equipped with a mosaic of four Hawaii 2 RG $2048 \times 2048$ pixel detectors with a scale of $0.106^{\prime \prime}$ per pixel. The camera has a field of view on the sky of $7.5^{\prime} \times 7.5^{\prime}$ with a small cross-shaped gap of $\sim 15^{\prime \prime}$ between the four detectors. The observations of the CNC were performed from 24 to 31 January 2008 in service mode as part of the scientific verification program for HAWK-I. In addition to the standard broadband $J, H$, and $K_{\mathrm{S}}$ filters, we used the $\mathrm{H}_{2}(2.109-2.139 \mu \mathrm{m})$ and $\operatorname{Br} \gamma(2.150-2.181 \mu \mathrm{m})$ narrow-band filters.

Our survey consists of a mosaic of 24 contiguous HAWKI fields, covering a total area of about 1280 square-arcminutes; 
T. Preibisch et al.: Deep wide-field near-infrared survey of the Carina Nebula

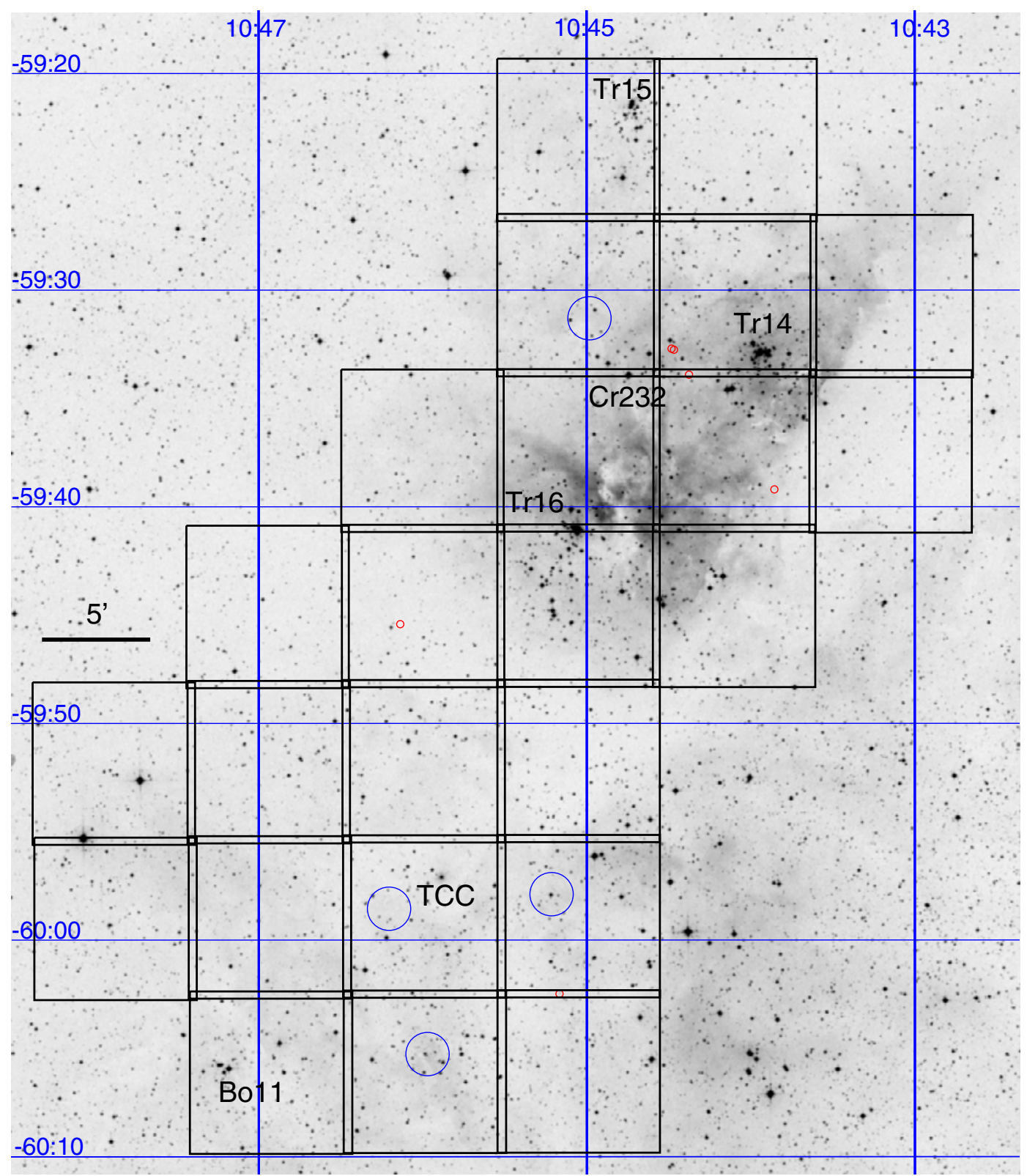

Fig. 1. Negative gray scale representation of an optical image of the Carina Nebula taken from the red Digitized Sky Survey plates, with superposed outline of the HAWK-I mosaic pattern. The individual HAWK-I mosaic fields are shown as boxes with a size of $7.5^{\prime} \times 7.5^{\prime}$. The clusters Tr 14 , $\operatorname{Tr} 15, \operatorname{Tr} 16, \mathrm{Cr} 232$, Bo 11, and the Treasure Chest Cluster (TCC) are marked. The $2^{\prime}$ diameter blue circles mark the positions of the four embedded clusterings discussed in Sect. 6, and the six small red circles indicate the positions of the six Molecular Hydrogen Emission Objects discussed in 7.1. North is up and east to the left. A grid of J2000 coordinates is also shown.

it includes the central part of the Nebula with $\eta$ Car and $\operatorname{Tr} 16$, the clusters $\operatorname{Tr} 14$ and Tr 15, and large parts of the South Pillars (see Fig. 1).

The total observing times for each mosaic position were 12,8 , and $5 \mathrm{~min}$ in $J, H$, and $K_{\mathrm{s}}$. Additional $5 \mathrm{~min}$ were spent with each of the $\mathrm{H}_{2}$ and $\mathrm{Br} \gamma$ narrow band filters. In order to account for the gaps of the detector array, we used a 5point dither pattern with offsets of $(0,0),\left(-40^{\prime \prime},+40^{\prime \prime}\right),\left(-40^{\prime \prime}\right.$, $\left.-40^{\prime \prime}\right),\left(+40^{\prime \prime},-40^{\prime \prime}\right)$, and $\left(+40^{\prime \prime},+40^{\prime \prime}\right)$ at each individual mosaic position. Details of the observing parameters are listed in Table 1.

Thanks to the very good seeing conditions during the observations, most of the HAWK-I images are of a very high image quality. The $F W H M$ of the Point-Spread-Function in the HAWKI images is typically $0.6^{\prime \prime}-0.8^{\prime \prime}$. This good image quality led to
Table 1. Observing parameters.

\begin{tabular}{lcccc}
\hline \hline Filter & Offsets & Integrations & $\begin{array}{c}\text { Integration } \\
\text { times }[\mathrm{s}]\end{array}$ & $\begin{array}{c}\text { Total exposure } \\
\text { time }[\mathrm{s}]\end{array}$ \\
\hline$J$ & $2 \times 5$ & 24 & 3 & 720 \\
$H$ & $2 \times 5$ & 16 & 3 & 480 \\
$K_{\mathrm{s}}$ & 5 & 20 & 3 & 300 \\
$\mathrm{H}_{2}$ & 5 & 2 & 30 & 300 \\
$\mathrm{Br} \gamma$ & 5 & 2 & 30 & 300 \\
\hline
\end{tabular}

the detection of interesting structures such as the edge-on circumstellar disk described in Preibisch et al. (2011c).

As examples for the quality of the HAWK-I data, we show comparisons of the HAWK-I and 2MASS images of selected regions in the CNC in Fig. 2. 


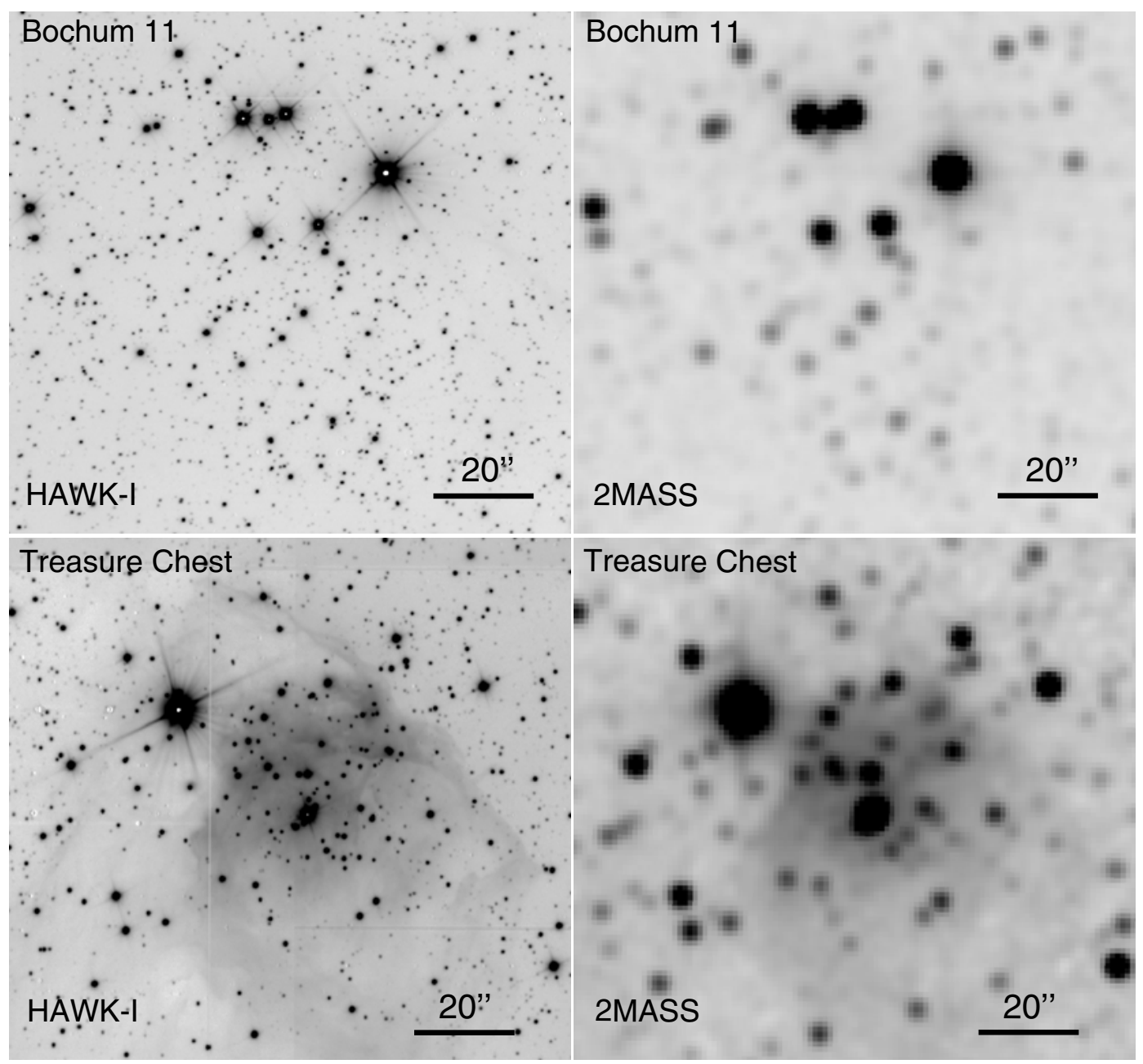

Fig. 2. Comparison of HAWK-I (left) and 2MASS (right) $H$-band images of Bochum 11 (above) and the Treasure Chest cluster (below).

\subsection{Photometry and construction of the HAWK-I source catalog}

The procedures performed to derive photometry from the HAWK-I images and the construction of the source catalog is described in detail in Preibisch et al. (2011b). Here we briefly summarize the main points. All HAWK-I data were processed and calibrated by the Cambridge Astronomical Survey Unit using pipeline software as described in Irwin et al. (2004). A photometric analysis was performed for all images obtained in the three broad-band $J, H$, and $K_{\mathrm{s}}$, following closely the procedures described in detail in Hodgkin et al. (2009). The photometric calibrators are drawn from stars in the 2MASS Point Source Catalog, which are present in large numbers (several hundred) in each HAWK-I mosaic field. The source lists of the individual mosaic positions were then combined into total catalogs (per band) by removing duplicates from the overlap regions; this was achieved by keeping the source with the highest signal-to-noise ratio. The final catalog was generated by merging the source catalogs for the individual bands into a combined catalog. The maximum allowed spatial offset for inter-band identifications of individual objects was set to $\leq 0.3^{\prime \prime}$.

The final HAWK-I photometric catalog contains 600336 individual objects. Most (502714) catalog objects are simultaneously detected in the $J$-, $H$-, and the $K_{\mathrm{s}}$-band. Objects as faint as $J \sim 23, H \sim 22$, and $K_{\mathrm{s}} \sim 21$ are detected with $S / N \geq 3$. Typical values for the completeness limit across the field are $J_{\text {compl }} \sim 21$,
$H_{\text {compl }} \sim 20$, and $K_{\text {s, compl }} \sim 19$; nearly all objects above these limits are $S / N \geq 10$ detections. Our survey is thus about 5 mag deeper than 2MASS and represents the largest and deepest NIR survey of the CNC obtained so far.

The absolute photometric accuracy of the catalog was characterized by a comparison of the HAWK-I and 2MASS photometry for a sample of more than 700 stars. The standard deviations between HAWK-I and 2MASS magnitudes and colors are found to be $\sigma_{J}=0.11 \mathrm{mag}, \sigma_{H}=0.08 \mathrm{mag}, \sigma_{K_{\mathrm{s}}}=0.11 \mathrm{mag}$, $\sigma_{J-H}=0.12 \mathrm{mag}$, and $\sigma_{H-K_{\mathrm{s}}}=0.11 \mathrm{mag}$. The accuracy is mainly limited by the photometric quality of the 2MASS stars suitable for comparison and the strong and highly variable diffuse nebulosity in the area of the HAWK-I mosaic.

\subsection{Background contamination in the HAWK-I catalog}

Due to the CNC's location almost exactly on the galactic plane, the degree of field star contamination is very high. This causes a fundamental limitation of the usefulness of the infrared source catalog. The degree of contamination was recently quantified in two Spitzer studies of the CNC. Smith et al. (2010b) and Povich et al. (2011a) found that only $\approx 3 \%$ of the $\sim 50000$ Spitzer infrared sources in the CCCP survey area can be classified as candidate YSOs. The contamination rate of our deep NIR HAWK-I data is presumably similarly high. This suggests that the vast majority ( $\gtrsim 90 \%)$ of the $\sim 600000$ detected infrared sources in our 


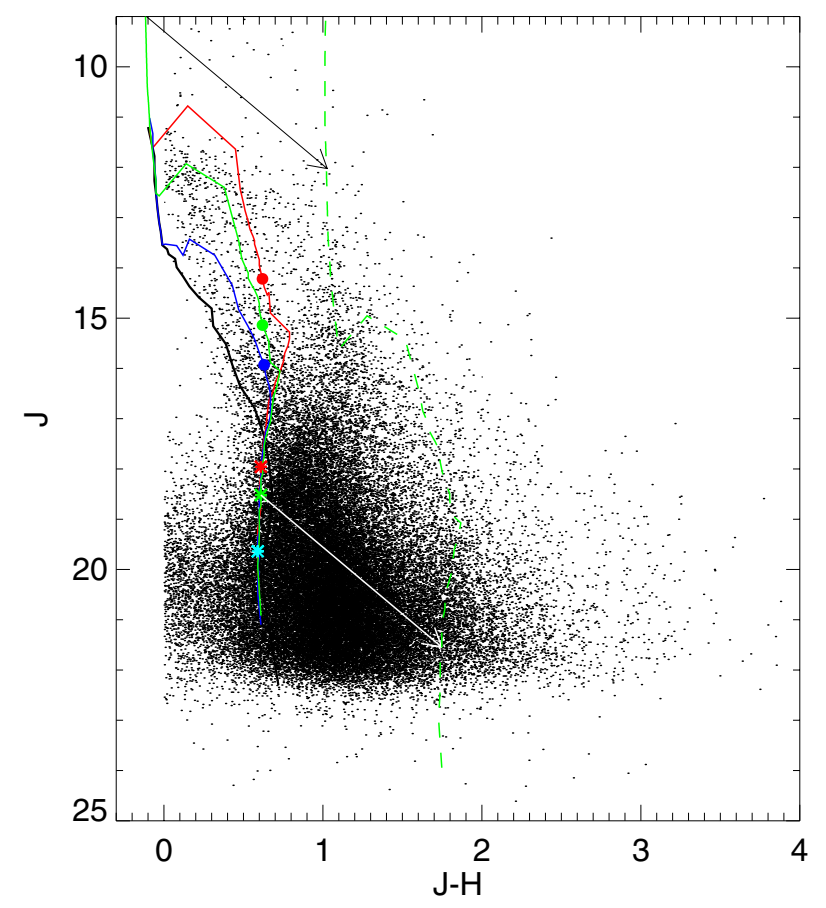

Fig. 3. Color-magnitude diagram of all HAWK-I sources with NIR excesses. The solid lines show isochrones for ages of $1 \mathrm{Myr}$ (red), $3 \mathrm{Myr}$ (green), $10 \mathrm{Myr}$ (blue), and the ZAMS composed from the models of Baraffe et al. (1998) for the mass range 0.02 to $0.5 M_{\odot}$ and Siess et al. (2000) for the mass range 0.5 to $7 M_{\odot}$. The large solid dots mark the positions of $1 M_{\odot}$ stars, the asterisks those of $0.075 M_{\odot}$ objects on the 1,3 , and $10 \mathrm{Myr}$ isochrones. The arrows indicate reddening vectors for $A_{\mathrm{V}}=10$ mag and were computed for an extinction law with $R_{\mathrm{V}}=4$ (see Povich et al. 2011b). The dashed green line shows the $3 \mathrm{Myr}$ isochrone reddened by that amount. Note that all bright objects with $J<12.5$ are in the saturation/non-linearity regime of the HAWK-I data and their photometry is therefore unreliable.

HAWK-I catalog must be foreground or background sources, unrelated to the $C N C$.

\subsection{Contamination and incompleteness of NIR excess selected samples}

This high degree of contamination requires a very efficient and reliable selection method to identify YSOs among the very numerous contaminants. A widely used method is to select objects with infrared excess emission, which is a tracer of circumstellar material, as YSO candidates. In order to test whether such an approach could be useful for our data, we analyzed the $J-H$ vs. $H-K_{\mathrm{s}}$ color-color diagram of the HAWK-I sources. Objects are classified as NIR excess sources if they lie at least $\geq 1 \sigma_{\text {phot }}$ and more than 0.05 mag to the right and below the reddening band, based on the intrinsic colors of dwarfs (Bessel \& Brett 1988) and an extinction vector with slope 1.73, and above $J-H=0$. In this way, 50950 of the 502714 HAWK-I sources with complete $J, H$, and $K_{\mathrm{s}}$ photometry are classified as NIR excess sources. The color-magnitude diagram (CMD) of these NIR excess sources is shown in Fig. 3.

However, there are two reasons why we think that this excess-selected sample is not a useful sample of the YSOs in the CNC. First, due to the very high level of background contamination, even an excess selected sample will still be highly polluted by background objects. Several recent spectroscopic studies of infrared sources in other star forming regions have shown that excess-selected YSO candidate samples can be strongly contaminated by background sources such as evolved Be stars, carbon stars, or planetary nebulae, which often show NIR colors very similar to those of YSOs (e.g., Mentuch et al. 2009; Oliveira et al. 2009; Rebull et al. 2010). Our CMD suggests this to be the case here too: a large majority $(78 \%)$ of the NIR excess objects is found at very faint magnitudes $(J>19)$. Comparing the location of the sources in the CMD to pre-main sequence models shows that $61 \%$ of all NIR excess objects are below the expected reddening vector for $3 \mathrm{Myr}$ old $0.075 M_{\odot}$ objects in Carina. While some of these faint objects may be deeply embedded YSOs, stars with edge-on circumstellar disks, or disk-bearing young brown dwarfs, the majority are very likely not young stars in the CNC but background objects. Furthermore, we note that the HAWK-I images are deep enough to detect numerous extragalactic objects. Since star-forming galaxies and AGN often display NIR colors similar to those of YSOs, this leads to even higher contamination rates in NIR excess selected samples of YSO candidates $^{1}$.

The second problem of a NIR excess selected YSO candidate sample is its incompleteness. It is well known that NIR excess emission in young stars disappears on timescales of just a few Myr (e.g., Briceño et al. 2007); at an age of $~ 3 \mathrm{Myr}$, only $\sim 50 \%$ of the young stars still show NIR excesses, and by $\sim 5$ Myr this is reduced to $\sim 15 \%$. Since the expected ages of most young stars in the CNC are several Myr, any excess-selected YSO sample will be highly incomplete.

Considering these problems, we conclude that due to the very high degree of background contamination and the high expected level of incompleteness of NIR excess selected YSO candidate samples, the NIR data alone cannot provide a reasonably clean and complete sample of YSOs. Without additional information, the HAWK-I data alone can yield only very limited insight into the young stellar populations in the CNC.

\subsection{Combination of infrared and $X$-ray data}

Sensitive X-ray observations can provide a very good solution for the problem of identifying the young stars in an extended complex such as the CNC. X-ray surveys detect the young stars by their strong X-ray emission (e.g., Feigelson et al. 2007) and efficiently discriminate them from the numerous older field stars in the survey area. X-rays are equally sensitive to young stars which have already dispersed their circumstellar disks, thus avoiding the bias introduced when selecting samples based only on infrared excess. Many X-ray studies of star forming regions have demonstrated the success of this method (see, e.g., Preibisch et al. 1996; Preibisch \& Zinnecker 2002; Broos et al. 2007; Forbrich \& Preibisch 2007; Wang et al. 2010). Also, the relations between the X-ray properties and basic stellar properties in young stellar populations are now very well established from very deep X-ray observations such as the Chandra Orion Ultradeep Project (see Getman et al. 2005; Preibisch et al. 2005).

The combination of the HAWK-I infrared source catalog with the list of X-ray sources detected was an essential aspect of the Chandra Carina Complex Project (CCCP, see Townsley et al. 2011). The details of the infrared - X-ray source matching

\footnotetext{
${ }^{1}$ Based on the number counts of star-forming galaxies in the data from the UKIRT Infrared Deep Sky Survey data by Lane et al. (2007) we can expect the presence of about 4700 star-forming galaxies with $K_{\mathrm{s}} \leq 20$ in the HAWK-I field. From a purely statistical point-of-view, all of the 2157 NIR excess objects with $K_{\mathrm{s}}>20$ in the HAWK-I catalog may well be extragalactic sources.
} 
is described in Preibisch et al. (2011b), and here we just briefly summarize the main aspects: The area of the HAWK-I mosaic covers $27 \%$ of the CCCP survey area and contains $52 \%$ of all 14368 detected X-ray sources. The HAWK-I infrared catalog provided infrared matches for 6583 of these X-ray sources. After adding matches from the 2MASS catalog for very bright stars that are saturated in the HAWK-I images, infrared counterparts were established for $6636 \mathrm{X}$-ray sources. 6241 of these have valid photometry in all three of the $J_{-}, H$-, and $K_{\mathrm{s}}$-bands, and $6173(93.0 \%)$ are classified as Carina members (see Broos et al. 2011b).

Using the NIR photometry of the X-ray selected Carina members, it was found that the typical extinction values for the diskless stars range from $A_{\mathrm{V}} \sim 1.6 \mathrm{mag}$ to $\sim 6.2 \mathrm{mag}$ (central $80 \%$ percentile). While this clearly shows a considerable range of differential extinction between individual stars in the complex, it also implies that the vast majority of the X-ray detected young stellar population in the CNC shows rather moderate extinction, $A_{\mathrm{V}} \leq 10 \mathrm{mag}$. The $\mathrm{X}$-ray selected Carina members can thus be considered as a "lightly obscured" population of young stars. As will be discussed in Sect. 8, deep Spitzer data suggest the presence of an additional (but smaller) population of embedded YSOs which are not detected in the CCCP X-ray data.

\section{Color-magnitude diagrams for selected clusters in the CNC}

The stars in the CNC are distributed in a number of clusters (most notably the prominent optical clusters Tr 14, 15, and 16) and a widely dispersed population of young stars. In this section we consider the properties of the stellar populations in these different parts of the CNC. In order to avoid the background contamination problem, we restrict our analysis to the X-ray selected members, using the results of the clustering analysis of Feigelson et al. (2011).

In our analysis of the CMDs we use the isochrones derived from the pre-main sequence models of Baraffe et al. (1998) for the mass range 0.02 to $0.5 M_{\odot}$ and those of Siess et al. (2000) for the mass range 0.5 to $7 M_{\odot}$. For the more massive stars, which are on the main sequence or already in their post-main sequence phases, we used the Geneva stellar models from Lejeune $\&$ Schaerer (2001) (model iso-c020-0650) for the mass range 7 to $70 M_{\odot}$.

Before we consider the individual CMDs, we want to point out that there are fundamental limitations to the accuracy of stellar mass- and age-determinations from CMDs (or HRDs); different studies of the same stellar population can sometimes produce severely discrepant results (see discussion in Hartmann 2001, 2003), especially with respect to the presence or absence of age spreads. Factors such as differential extinction, photometric variability, overestimates of the stellar luminosity due to unresolved binary companions, and the effects of accretion ${ }^{2}$ can cause substantial scatter in the diagram (see, e.g., discussion in Preibisch \& Zinnecker 1999; Slesnick et al. 2008). The observed apparent luminosity spreads in the data are easily mis-interpreted as age spreads (see discussion in Hillenbrand et al. 2008). It is therefore important to keep in mind that any observed luminosity spread in a CMD (or HRD) is always only an upper limit to a possible age spread. Underestimating the uncertainties in the observational

\footnotetext{
${ }^{2}$ Baraffe et al. (2009) showed that stars with intermittently variable accretion rates can produce a large spread of luminosities such that a population of stars with identical ages of a few Myr may be wrongly interpreted to have an age spread of as much as $\sim 10 \mathrm{Myr}$.
}

data can easily lead to biased results ${ }^{3}$. We will therefore not attempt to determine ages and masses for individual stars, but restrict our interpretation of the CMDs to estimates of the typical ages for the total population of young stars in each cluster; in a large enough sample, most of the above-mentioned uncertainties are expected to cancel out statistically. With these caveats in mind we now take a look at the CMDs for individual parts of the CNC.

\subsection{The cluster Trumpler 16}

The loose cluster $\operatorname{Tr} 16$ is located in the center of the Carina Nebula and includes the optically dominant star $\eta$ Car as well as the majority of the O-type stars in the CNC. All age estimates for $\operatorname{Tr} 16$ reported so far in the literature concern only the high- and intermediate mass stars. Massey et al. (2001) found that the highmass population of $\operatorname{Tr} 16$ seems to be co-eval and report an age of 1.4 Myr (note that they assumed a distance of $3.1 \mathrm{kpc}$ rather than the value of $2.3 \mathrm{kpc}$ we use here). DeGioia-Eastwood et al. (2001) claimed that the intermediate-mass stars in $\operatorname{Tr} 16$ have been forming continuously over the last $10 \mathrm{Myr}$, whereas the high-mass stars formed within the last 3 Myr. Dias et al. (2002) list an age of 5 Myr, whereas Tapia et al. (2003) suggested continuous star formation since $6 \mathrm{Myr}$ until now. These claims of large age spreads may be overestimates caused by the considerable differential reddening across the areas of these loose clusters. The most reliable age estimate seems to be the value of 3-4 Myr that was suggested by Smith \& Brooks (2008) based on detailed stellar evolution models for $\eta$ Car, the presence of $\mathrm{O} 3$ main sequence stars, and the modeling of the main-sequence turn-off in optical color-magnitude diagrams.

The CMD of Tr 16 shown in Fig. 4 is based on the 449 stars with complete $J$ - and $H$ - and $K_{\mathrm{s}}$-band photometry among the $529 \mathrm{X}$-ray sources that are classified as Carina members and associated to one of the sub-clusters in the $\operatorname{Tr} 16$ area by Feigelson et al. (2011). Concerning the age of the low-mass stellar population, we note that our CMD of the X-ray selected sources shows a conspicuous group of stars with $J \sim 12-14$ apparently aligned with the 3-4 Myr isochrones. The bulk of the low-mass stars is also seen at locations consistent with ages of $\approx 3-4 \mathrm{Myr}$, and thus we consider this to be the most likely age for the low-mass stellar population in $\operatorname{Tr} 16$. This value is consistent with the most likely age of the high-mass members, suggesting that high- and low-mass stars have formed at the same time. We note that a more comprehensive study of the X-ray selected population of $\operatorname{Tr} 16$ can be found in Wolk et al. (2011).

\subsection{The cluster Trumpler 14}

Tr 14 is the second most massive cluster in the Carina Nebula and contains 10 known O-type stars. Its spatial configuration is considerably more compact than $\operatorname{Tr} 16$. The published age estimates for Tr 14 show remarkable discrepancies: while DeGioiaEastwood et al. (2001) claim continuous star formation over the last 10 Myr, Dias et al. (2002) list an age of 2 Myr. Tapia et al. (2003) suggested a scenario of continuous star formation lasting since 5 Myr until less than 1 Myr ago. Ascenso et al. (2007)

\footnotetext{
3 To mention just two examples we refer to the study of the young cluster NGC 3293 by Baume et al. (2003), in which a careful analysis led to a correction of previous claims about the stellar ages, and to the new results of Currie et al. (2010) for $h$ and $\chi$ Persei, that clearly refute earlier claims of different ages and large age spreads for the double cluster.
} 

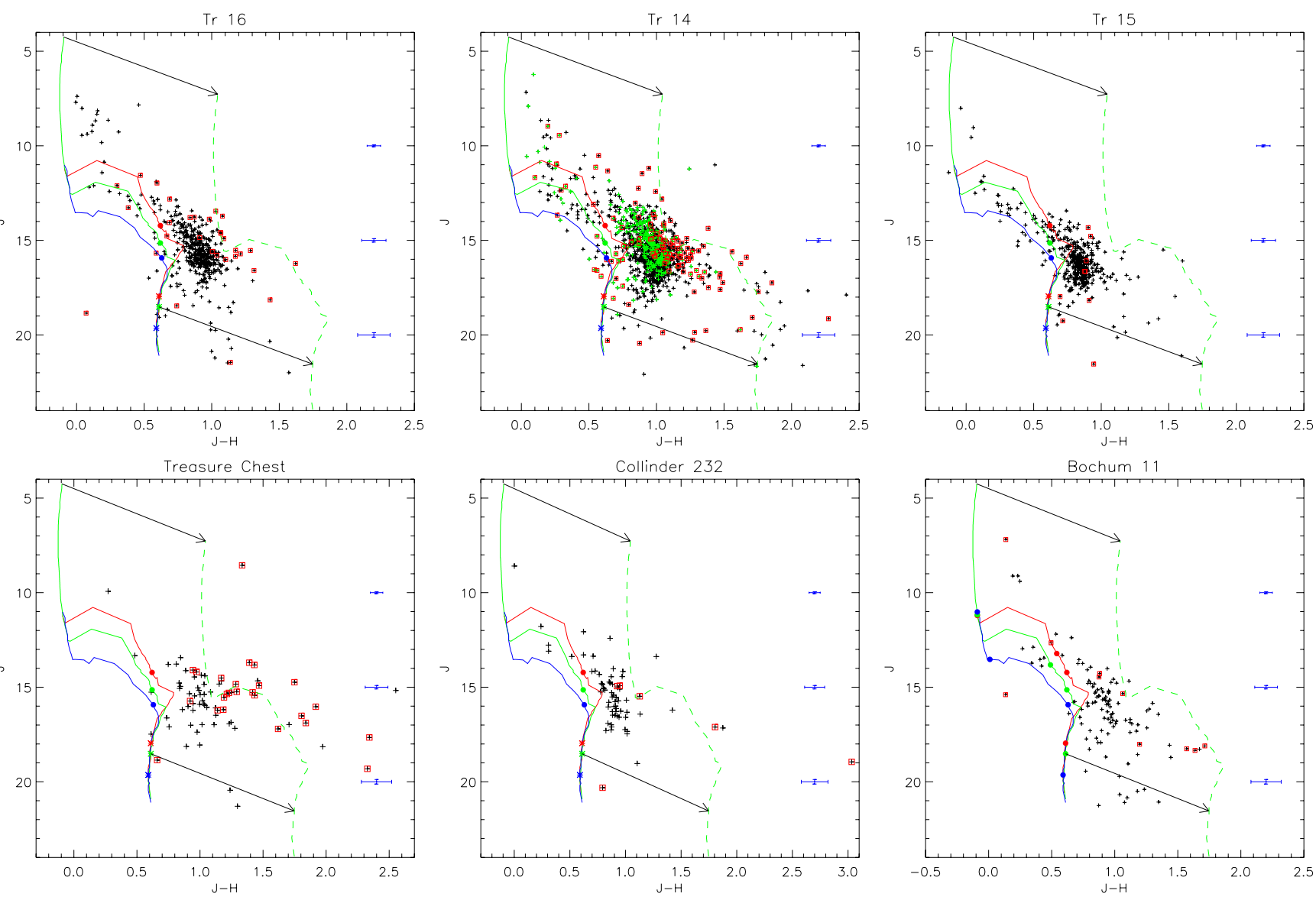

Fig. 4. Color-magnitude diagrams for the X-ray selected Carina members (crosses) in the different clusters in the CNC. Objects with infrared excesses are additionally marked by red open boxes. The solid green line shows the $3 \mathrm{Myr}$ isochrone, the red and the blue lines show the lowmass $\left(M \leq 7 M_{\odot}\right)$ isochrones for ages of $1 \mathrm{Myr}$ and $10 \mathrm{Myr}$, respectively. The large solid dots mark the positions of $1 M_{\odot}$ stars, the asterisks those of $0.075 M_{\odot}$ objects on these isochrones. The arrows show the extinction vector for $A_{\mathrm{V}}=10 \mathrm{mag}$, and the dashed green line shows the $3 \mathrm{Myr}$ isochrone redenned by that amount. The sequence of blue errorbars near the right edge indicates the typical range of magnitude dependent photometric uncertainties. In the CMD for $\operatorname{Tr} 14$, the green crosses mark the X-ray sources in the central $R=2^{\prime}$ cluster core.

claimed a more or less continuous age distribution between zero and $\sim 5 \mathrm{Myr}$, and suggested three peaks in the age distribution at values of 0-0.3 Myr, 1.4-2.5 Myr, and 3.2-5 Myr. Sana et al. (2010) analyzed new adaptive-optics images of the cluster core (radius $=2^{\prime}$ ) and derived a very young cluster age of only $\sim 0.3-0.5 \mathrm{Myr}$.

Our CMD of Tr 14 (Fig. 4) is based on the membership to the rather extended X-ray cluster as revealed by Feigelson et al. (2011). It contains 1378 Chandra sources, for 1219 of which we have complete NIR photometry. The CMD suggests a somewhat broader age distribution than for $\operatorname{Tr} 16$. Some objects lie between the 3 Myr and 10 Myr isochrones, but we note that most of these apparently older objects are located in the outer parts of the cluster. If we restrict the sample to the $R=2^{\prime}$ cluster core, most of the apparently older objects are removed. The tendency for younger stellar ages in the cluster center agrees with the recent results from Sana et al. (2010). The bulk of the stars in the cluster center seem to be quite young, $\lesssim 3 \mathrm{Myr}$. Since this value is in reasonable agreement with the age estimate for the high-mass stars in Tr 14 by Dias et al. (2002), we conclude that low- and high-mass stars in Tr 14 seem to be coeval. Many stars in the outer parts of the cluster seem to be somewhat older; however, it remains unclear whether these stars in the outer regions are actually members of the cluster $\operatorname{Tr} 14$, or whether they may be part of the widely distributed population of (partly older) stars in the CNC.

\subsection{The cluster Trumpler 15}

The cluster Tr 15 is located in the northern part of the CNC and contains 6 O-type stars. It is thought to be several Myr older than $\operatorname{Tr} 16$ and Tr 14. Dias et al. (2002) list an age of 8 Myr, while Tapia et al. (2003) claim a range of ages between $\sim 4$ Myr and $\sim 30 \mathrm{Myr}$, with a median value of about $8 \mathrm{Myr}$ for the high-mass stars in $\operatorname{Tr} 15$.

Our CMD (Fig. 4) is again based on the membership analysis of Feigelson et al. (2011). It contains 481 Chandra sources, for 436 of which we have NIR photometry. The upper part of the CMD is dominated by a group of stars lying between the $3 \mathrm{Myr}$ and the $10 \mathrm{Myr}$ isochrones; together with the clear lack of bright objects to the right of the $3 \mathrm{Myr}$ isochrone, this suggests an age of 2-8 Myr for the low-mass stars. This agrees with the age derived for the high-mass stars in $\operatorname{Tr} 15$, suggesting that highand low-mass stars are co-eval, and clearly showing that $\operatorname{Tr} 15$ is older than $\operatorname{Tr} 16$ and $\operatorname{Tr} 14$. Finally, we note that a comprehensive study of the X-ray selected population of Tr 15 can be found in Wang et al. (2011).

\subsection{The "Treasure Chest" cluster}

The most prominent of the embedded clusters in the Southern Pillars region is the "Treasure Chest", which is thought to be very young ( $<1 \mathrm{Myr}$, see Smith et al. 2005). The most 
massive member is a $09.5 \mathrm{~V}$ star. The $\mathrm{X}$-ray clustering analysis of Feigelson et al. (2011) associated 96 Chandra sources to the Treasure Chest. The 78 sources for which we have NIR photometry are plotted in Fig. 4. The typical extinction of the member stars, $A_{\mathrm{V}} \geq 5 \mathrm{mag}$, is considerably larger than in the other regions. The CMD of the X-ray selected stars is consistent with a very young age, since nearly all stars lie well above the $1 \mathrm{Myr}$ isochrone.

\subsection{The cluster Collinder 232}

The clustering Cr 232 is located about $3^{\prime}$ to the east of $\operatorname{Tr} 14$ and defined by the early type stars HD 93250 (O3.5V), HD 303311 (O5), and HD 93268 (A2). Until recently, the reality of a stellar cluster was not clear. Tapia et al. (2003) noted that Cr 232 may be just a random coincidence of these three relatively bright stars. However, the spatial distribution of the X-ray detected young stars in the CCCP clearly shows a significant density peak in the Cr 232 region (Feigelson et al. 2011). There are 70 Chandra sources associated with this peak, for 67 of which we have $J+H+K_{\mathrm{s}}$ photometry. The CMD is consistent with the assumption that the majority of the stellar population in this region is very young, $\$ 3 \mathrm{Myr}$.

Our HAWK-I images reveal several very red, deeply embedded infrared sources in $\mathrm{Cr} 232$, including the prominent circumstellar disk object discussed in more detail in Preibisch et al. (2011c). This detection of embedded infrared sources, and the jets discussed in Sect. 7 clearly shows that Cr 232 actually is a cluster of very young stars and with ongoing star formation activity. We note that the IRAS and MSX data also led to the detection of deeply embedded luminous infrared sources in $\mathrm{Cr} 232$ (e.g., Mottram et al. 2007), some of which may be relatively massive YSOs.

\subsection{The cluster Bochum 11}

Bochum 11 (see Fig. 2) is a loose open cluster in the southeastern part of the CNC (i.e. in the South Pillars region) and contains 5 O-type stars. From optical color-magnitude diagrams, age estimates of $\leq 3 \mathrm{Myr}$ (Fitzgerald \& Mehta 1987) and $~ 6$ Myr (Dias et al. 2002) for the high-mass stars have been published.

The X-ray clustering analysis associated 136 Chandra sources to Bochum 11. The CMD of the $117 \mathrm{X}$-ray selected stars with available NIR photometry (Fig. 4) shows a group of stars lying between the $3 \mathrm{Myr}$ and the $10 \mathrm{Myr}$ isochrones. This suggests that Bo 11 is somewhat older than $\operatorname{Tr} 14$ and $\operatorname{Tr} 16$, probably around 5 Myr. This agrees reasonably well with the age estimate for the high-mass stars from Dias et al. (2002).

\subsection{The "widely distributed" population}

According to the clustering analysis of Feigelson et al. (2011), 5185 of the 10714 X-ray detected Carina members are not associated with any cluster and thus constitute a widely dispersed population of young stars. Note that due to the spatial sensitivity variations (caused by the off-axis mirror vignetting and degradation of the point spread function), the X-ray detection completeness for this dispersed population is somewhat lower than for the previously known clusters (that were preferentially observed at small on-axis angles in the CCCP survey mosaic). This implies that the relative size of this dispersed population (compared to the X-ray detected populations of the clusters) is somewhat underestimated.

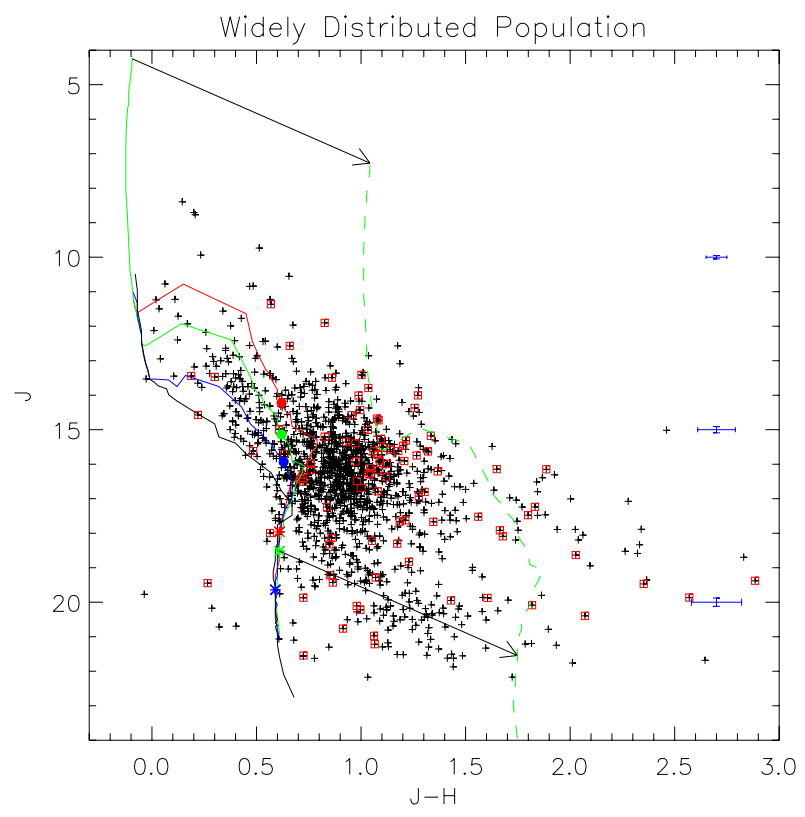

Fig. 5. Color-magnitude diagram for the X-ray selected Carina members in the "widely distributed population" within the HAWK-I survey area. The meaning of the symbols is as in Fig. 4.

For 1412 of these objects in the HAWK-I survey area we have complete $J$ - and $H$ - and $K_{\mathrm{s}}$-band photometry. The CMD of these distributed stars (Fig. 5) is clearly different from the CMDs of the clusters $\operatorname{Tr} 14,15$, and 16, because it shows a much broader spread of colors. The considerable number of objects to the left of the $3 \mathrm{Myr}$ isochrone and several objects near or to the left of the 10 Myr isochrone suggest a rather broad age distribution. We also note that the relative number of bright objects is considerably smaller than seen in the CMDs of the clusters; this suggests that the fraction of high-mass stars is smaller in the distributed population compared to the clusters.

\section{NIR excess fractions and ages of the different clusters in the CNC}

The fraction of X-ray selected stars with $J H K$ excess emission has been determined in Preibisch et al. (2011b) to be $(9.7 \pm 0.8) \%$ in $\operatorname{Tr} 14,(2.1 \pm 0.7) \%$ in $\operatorname{Tr} 15,(6.9 \pm 1.2) \%$ in $\operatorname{Tr} 16$, and $(32.1 \pm 5.3) \%$ in the Treasure Chest. For Bochum 11 we find that 10 of the 117 sources with NIR photometry have NIR excesses, giving an excess fraction of $(8.5 \pm 2.6) \%$. Here we consider the relation between these excess fractions and the ages of the individual clusters derived from the analysis of the CMDs as described above. Figure 6 shows our results for the clusters in the $\mathrm{CNC}$ and compares it to similarly determined $J H K$ excess fractions in other galactic clusters.

It is obvious that the NIR excess fractions for all the clusters in Carina are systematically and considerably lower (by a factor of about two) than those for the other young galactic clusters of similar ages. We interprete this difference as a consequence of the very harsh conditions due to massive star feedback in the CNC. The other clusters used for comparison contain much smaller numbers of massive stars and are thus characterized by much more quiescent conditions. The large population of very massive stars in the CNC leads to a level of hydrogen ionizing flux that is about 150 times higher than in the Orion Nebula (Smith \& Brooks 2008); the resulting increased heating and photoevaporation of the circumstellar disks around low-mass stars in 


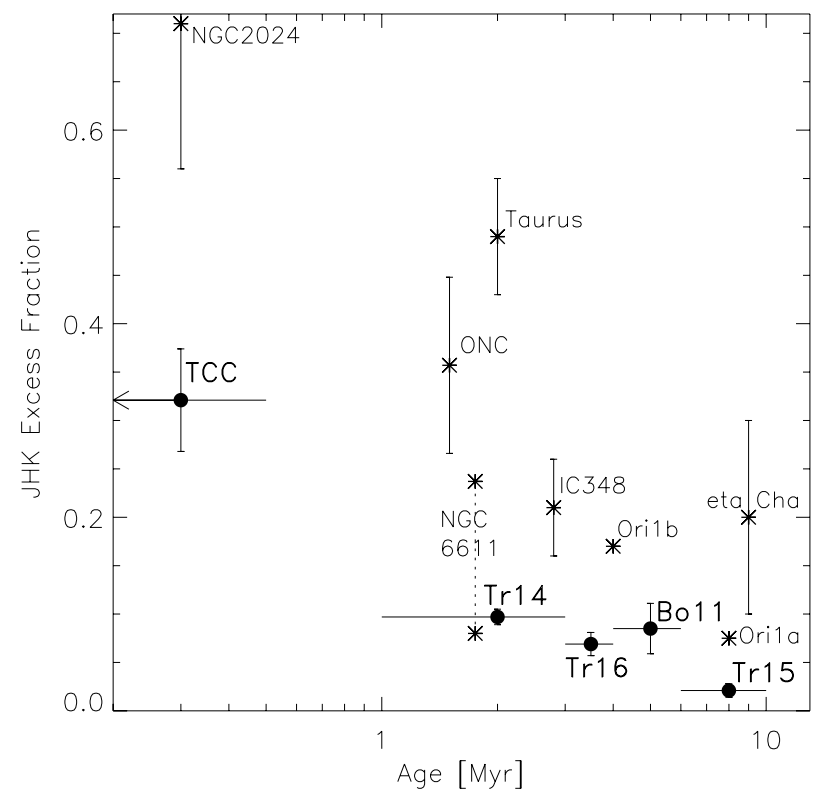

Fig. 6. $J H K$ excess fraction versus age for the clusters in the CNC. For comparison, we also show JHK excess fractions derived for NGC 2024 and Taurus (Haisch et al. 2000), the Orion Nebula Cluster (ONC, Wolk et al. 2005), IC 348 (Haisch et al. 2001), eta Cha (Lyo et al. 2003), Ori OB 1a and 1b (Briceño et al. 2005). For the case of NGC 6611, we plot the spatial variation of excess fractions from the center of the cluster to the outer parts as derived by Guarcello et al. (2007).

the $\mathrm{CNC}$ is expected to lead to a quicker dispersal of these disks (e.g., Clarke 2007).

This interpretation is supported by results about the spatial variation of the NIR excess fraction in the young cluster NGC 6611, that also contains a considerable population of massive stars in the center. Guarcello et al. (2007) found that the excess fraction of the X-ray detected low-mass stars is correlated with the distance from the massive stars and drops from $24 \%$ for the outer parts of the cluster to $8 \%$ in the center (i.e. close to the massive stars). This variation agrees well with the difference in the excess fraction between the $\mathrm{CNC}$ clusters and the other galactic clusters of similar ages.

\section{The size of the stellar population in the CNC}

Our infrared data of the X-ray selected CNC members provide information about the masses of these stars that can be used to infer properties of the stellar mass function in the CNC. For this, we first have to consider the X-ray detection completeness. The study of the CCCP data by Broos et al. (2011a) shows that the completeness limit for lightly obscured stars observed at smallto-moderate off-axis angles on the detector is $L_{X} \approx 10^{30} \mathrm{erg} / \mathrm{s}$ in the $0.5-8 \mathrm{keV}$ band. Assuming that the young stars in the CNC follow the relation between stellar mass and X-ray luminosity established by the data from the Chandra Orion Ultradeep Project (see Preibisch et al. 2005), we can expect a detection completeness of $\geq 80 \%$ for stars with $M_{*} \geq 1 M_{\odot}$. This completeness drops towards lower masses and should become $\$ 50 \%$ for $M_{*} \leq 0.5 M_{\odot}$.

We can thus assume the sample of X-ray selected Carina members to be nearly complete for stellar masses $M_{*} \geq 1 M_{\odot}$. In order to determine the observed number of stars above this mass limit, we used the CMD for the X-ray selected Carina members in the HAWK-I survey area. We find 3182 objects at CMD positions corresponding to $M_{*} \geq 1 M_{\odot}$ for an assumed age of $3 \mathrm{Myr}$ (i.e. above the reddening vector originating from the predicted position of 3 Myr old $1 M_{\odot}$ stars).

It is interesting to compare this number to an IMF extrapolation based on the known population of high-mass stars in the CNC. The compilation of Smith (2006) lists 127 individually identified members with known spectral types, and 52 of these should have stellar masses $M \geq 20 M_{\odot}$ according to the calibrations of Martins et al. (2005). The recent study of Povich et al. (2011b) lead to the detection of further candidates for young high-mass stars in the $\mathrm{CNC}$ and suggests that the true number of high-mass stars should be about $\sim 50 \%$ larger than the known population. Assuming thus a number of $\approx 78$ stars with $M \geq 20 M_{\odot}$, we can use the model representation of the field star IMF by Kroupa (2002) and find that the predicted total population of stars with $M_{*} \geq 1 M_{\odot}$ is $\approx 4400$. Since $80 \%$ of the known high-mass stars are inside the HAWK-I survey area, we thus have to expect that $\approx 3500$ stars with $M_{*} \geq 1 M_{\odot}$ should be found within the HAWK-I survey area, if the IMF in the CNC follows the Kroupa field star IMF.

This prediction agrees very well to the number of $3182 \mathrm{X}$-ray detected stars with CMD locations corresponding to $M_{*} \geq 1 M_{\odot}$. This agreement directly shows that the number of X-ray detected low-mass stars in the CNC is as high as expected from field IMF extrapolations of the high-mass stellar population. It is a direct confirmation that the IMF in the CNC is consistent with the field star IMF down to (at least) $M_{*} \approx 1 M_{\odot}$.

This conclusion is further supported by the finding that the shape of the K-band luminosity function of the X-ray selected Carina members agrees well with that derived for the Orion Nebula Cluster (Preibisch et al. 2011b). This implies that, down to the X-ray detection limit around $0.5-1 M_{\odot}$, the shape of the IMF in Carina is consistent with that in Orion (and thus the field IMF).

These results directly show that there is clearly no deficit of low-mass stars in the $C N C$ down to $\sim 1 M_{\odot}$. This is important because the issue whether the IMF is universal or whether there are systematic IMF variations in different environments is still one of the most fundamental open questions of star-formation theory (see Bastian et al. 2010, and references therein). It was often claimed that some (very) massive star forming regions have a truncated IMF, i.e. contain much smaller numbers of low-mass stars than expected from the field IMF. However, most of the more recent and sensitive studies of massive star forming regions (see, e.g., Liu et al. 2009; Espinoza et al. 2009) found the numbers of low-mass stars in agreement with the expectation from the "normal" field star IMF. Our result for the CNC confirms this and supports the assumption of a universal IMF (at least in our Galaxy). In consequence, this result also supports the notion that $\mathrm{OB}$ associations and very massive star clusters are the dominant formation sites for the galactic field star population, as already suggested by Miller \& Scalo (1978).

Based on this result, we can now proceed and make an estimate of the total stellar population (i.e. down to $\approx 0.1 M_{\odot}$ ) of the $\mathrm{CNC}$ by assuming that the X-ray luminosity function in the $\mathrm{CNC}$ is similar to that in Orion (as suggested by the data). The study of the CCCP source statistics by Feigelson et al. (2011) found that the 3220 bright X-ray sources with a photon flux of $\log (F) \geq-5.9$ photons $/ \mathrm{s} / \mathrm{cm}^{2}$ in the $0.5-8 \mathrm{keV}$ band constitute a spatially complete sample. If we transform this flux limit according to the difference in distances (i.e. $415 \mathrm{pc}$ for the ONC versus $2.3 \mathrm{kpc}$ for the $\mathrm{CNC}$ ), the comparison to the distribution of photon fluxes in the Chandra Orion Ultradeep Project sample (Getman et al. 2005) yields an estimate of $\sim 43727$ stars in total for the CNC. This number agrees very well with the 
extrapolation of the field IMF based on the number of massive stars (see above): for 78 stars with $M_{*} \geq 20 M_{\odot}$, the Kroupa IMF predicts that there should be $\approx 40000$ stars with $M_{*} \geq 0.1 M_{\odot}$.

Finally, we can multiply the extrapolated number of $\sim 43727$ stars with the mean stellar mass of $0.64 M_{\odot}$ (as valid for the Kroupa IMF in the $0.1 M_{\odot}$ to $100 M_{\odot}$ range), and find a total stellar mass of the inferred CNC population of about $28000 M_{\odot}$.

\section{Search for faint star clusters and results on the spatial distribution of the young stars}

The spatial distribution of the stars in the CNC contains important information about the structure, dynamics, and evolution of the region. A very important question in this context is whether the stars form in a clustered mode or in a dispersed mode. Until recently, only the high-mass part of the stellar population was well known. Most of the massive stars are in one of the open clusters (and this was the reason why the CNC was often considered to be a "cluster of clusters"). However, according to the field IMF, most of the total stellar mass is in the low-mass stellar population $^{4}$. Recent studies have shown that a significant fraction of all stars in the solar neighborhood seems to form in a nonclustered, dispersed mode (e.g., Gutermuth et al. 2009). Bressert et al. (2010) suggested that the stellar surface densities of young stars in nearby star forming regions follow one single smooth distribution rather than two different discrete modes (i.e. clustered versus distributed).

The spatial distribution and clustering properties of the X-ray detected YSOs in the CNC was studied in detail by Feigelson et al. (2011). They identified 20 principal clusters of X-ray stars (most of which correspond to known optical clusters in the CNC) and 31 small groups of X-ray stars outside the major clusters. Altogether, these clusters contain about half of the X-ray detected YSOs in the CNC. The other half of the X-ray detected YSO population seems to constitute a widely dispersed, but highly populous, distribution of more than 5000 X-ray stars. A similar result was found in the analysis of the Spitzer data: most of the $\sim 900$ identified YSO candidates are spread throughout the South Pillar region (Smith et al. 2010b). Although this Spitzer study led to the detection of eleven previously unknown clusters, the populations of these clusters are so small (at most $\leq 35$ YSOs per cluster) that only a small fraction of all YSOs are in one of these new clusters. While the analysis of the Chandra and Spitzer data yielded consistent results, the limited sensitivity of both data sets may leave some faint clusters undetected. The X-ray data are known to be seriously incomplete for masses $\lessgtr 0.5 M_{\odot}$. The same is true for the Spitzer data, that have a completeness limit of [3.6] 13, corresponding approximately to $M_{*}=1 M_{\odot}$. This implies that clusterings consisting of only a few dozen low-mass stars could have easily been missed. Such clusterings should, however, be easily visible in the HAWKI images, if their spatial configuration is compact enough. We have therefore performed a detailed visual inspection of the HAWK-I images to search for yet unrecognized clusters.

The very inhomogeneous cloud extinction causes strong spatial variations in the surface density of observed background objects in the HAWK-I images; this limits the ability to recognize loose clusterings consisting of only a small number $(\lessgtr 10)$ of stars. We therefore restricted the search to clusters that consist (apparently) of at least 10 stars and have a configuration

\footnotetext{
${ }^{4}$ According to the Kroupa IMF, 89\% of the total stellar mass in the $0.1-100 M_{\odot}$ range is contained in stars with $M_{*}<20 M_{\odot}$, and $73 \%$ in stars with $M_{*}<5 M_{\odot}$.
}

Table 2. Parameters of the newly detected clusters.

\begin{tabular}{lccccc}
\hline \hline Name & $R$ & $N_{*}$ & $N_{*, \text { exc. }}$ & $N_{*, \mathrm{X}}$ & $\begin{array}{c}\text { Brightest star } \\
K_{\mathrm{s}}[\mathrm{mag}]\end{array}$ \\
\hline [J2000] & {$\left[{ }^{\prime \prime}\right]$} & & & & 10.90 \\
C 104459-593118 & 11 & $\approx 33$ & 8 & 2 & 9.30 \\
C $104513-595753$ & 6 & $\approx 10$ & 1 & 4 & 10.60 \\
C 104559-600515 & 8 & $\approx 16$ & 3 & 2 & 12.86 \\
C 104613-595832 & 7 & $\approx 14$ & 8 & 2 & \\
\hline
\end{tabular}

Notes. We list the apparent radius $R$, the number of stars $N_{*}$, the number of NIR excess objects $N_{*, \text { exc. }}$, the number of X-ray detected stars $N_{*, \mathrm{X}}$ in each cluster, and the $K_{\mathrm{s}}$-band magnitude of the brightest star.

dense enough for them to clearly stand out from their surroundings. This search revealed only four likely clusters that were not known before. Images of these clusters are shown in Fig. 7 and their properties are listed in Table 2. We note that the physical nature of these apparent clusters is not entirely clear; some of them might be just random superpositions of unrelated objects at different distances. The cluster C 104559-600515 is the most reliable, because this group of stars appears to be embedded in the head of a prominent pillar in the South Pillars region. However, even in this case it remains unknown whether the apparent cluster is a gravitationally bound physical entity.

Despite the uncertainties about the physical reality of these clusters, our search provides a clear result: it directly confirms that there is no significant number of previously undetected clusters. The HAWK-I images clearly show that most of those young stars in the complex that are not associated to one of the already known clusters are in a non-clustered, dispersed spatial configuration. This result strongly supports the conclusions drawn from the X-ray clustering study of Feigelson et al. (2011) that about half of the total young stellar population is in a widely distributed spatial configuration. It also supports the picture of small-scale triggered star formation by radiative (and wind) feedback from the massive stars (see discussion in Sect. 8).

\section{Search for protostellar jets}

Jets and outflows are important signposts for embedded protostars that often remain undetected at NIR wavelengths, and can reveal the latest generation of the currently forming stars. When the outflowing material interacts with the environment, shocks are generated where the flows impact with surrounding dense clouds. In these shocks, hydrogen molecules can be collisionally excited into higher ro-vibrational levels and the subsequent decay of these excited states causes strong emission lines in the NIR wavelength range. The $2.12 \mu \mathrm{m} v=1-0 \mathrm{~S}(1)$ ro-vibrational emission line of molecular hydrogen is a very convenient and widely used tracer of these shocks (e.g., McCaughrean et al. 1994; Smith et al. 2007; Davis et al. 2008). This was the motivation for obtaining HAWK-I images through a narrow-band filter centered on the $2.12 \mu \mathrm{m} \mathrm{H}_{2}$ line.

It is, however, important to keep in mind that the detection of ro-vibrational line emission is not a proof of the presence of jetinduced shocks, because molecular hydrogen can also be excited in higher ro-vibrational levels by the mechanism of UV fluorescence (e.g., Black \& Dalgarno 1976). Since the Carina Nebula is characterized by very high levels of ionizing photon fluxes, UV fluorescence will be a very important excitation mechanism for $\mathrm{H}_{2}$ ro-vibrational emission. In our HAWK-I data, a distinction between collisional (shock) excitation and UV fluorescence can be made by comparing the strength of the emission seen in the $\mathrm{H}_{2}$ filter images to that in the images obtained through 


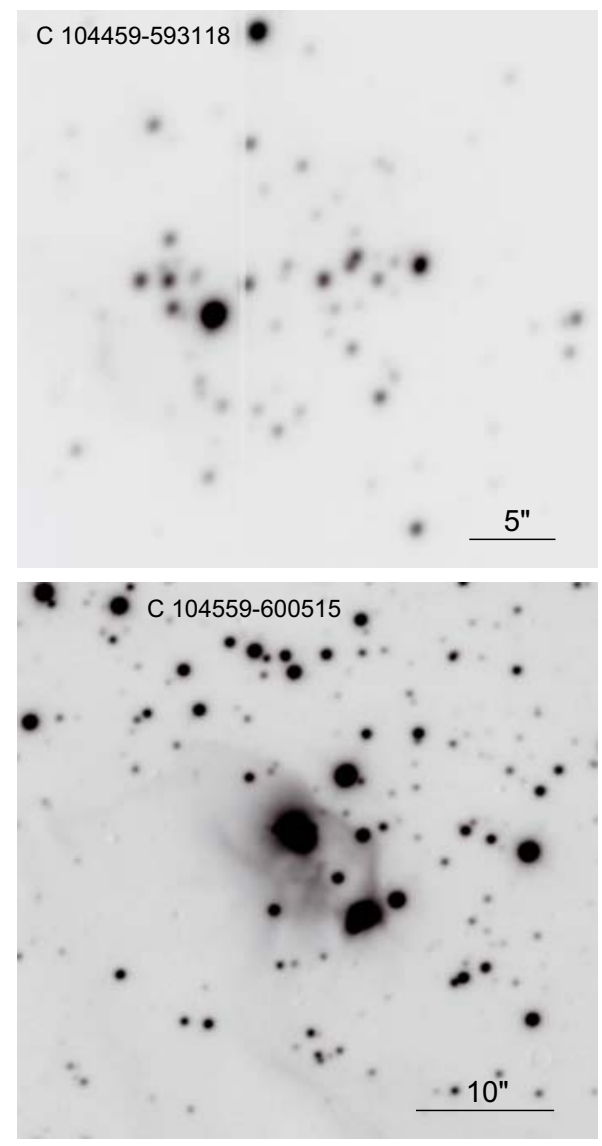

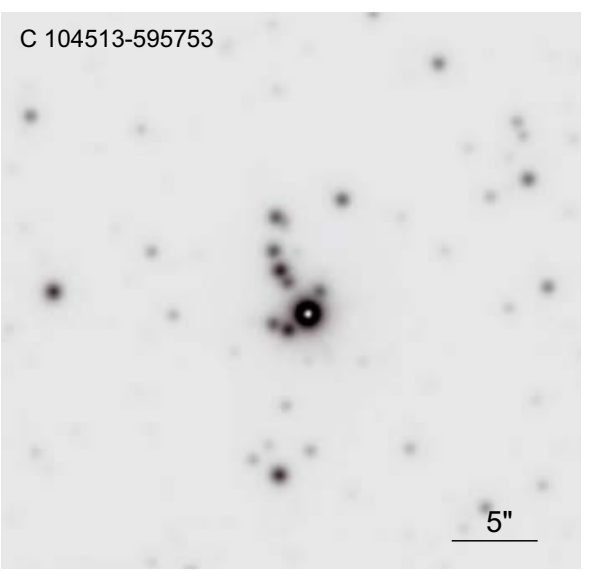

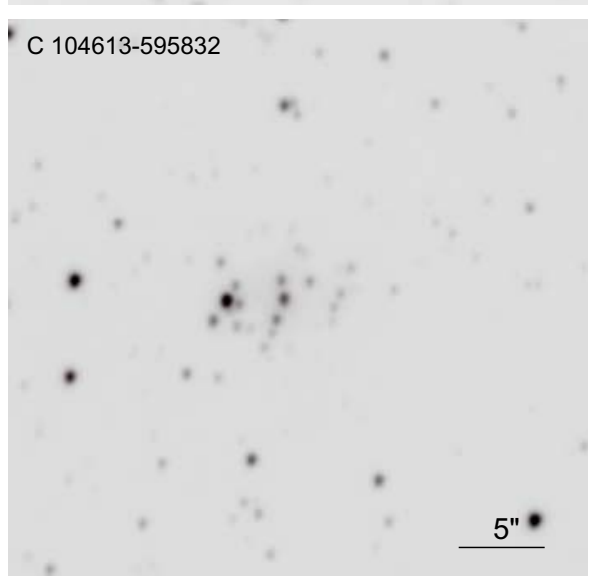

Fig. 7. HAWK-I $H$-band images of the four newly detected clusters. another narrow-band filter centered on the Br $\gamma$ line. Collisionally (jet-) excited molecular hydrogen from jets is not expected to show Bry emission (see, e.g., Nisini et al. 2002; Smith et al. 2007; Garcia Lopez et al. 2010), whereas UV excited (i.e. irradiated) matter should also show Br $\gamma$ emission.

\section{1. $\mathrm{H}_{2}$ jets in the HAWK-I images}

We performed a detailed visual inspection of the HAWK-I narrow-band images to search for features that are bright in the $\mathrm{H}_{2}$ filter and much fainter (or invisible) in the $\mathrm{Br} \gamma$ filter. This search revealed only six clear cases of Molecular Hydrogen Emission-Line Objects (MHOs). These objects have been included in the "Catalogue of Molecular Hydrogen EmissionLine Objects in Outflows from Young Stars" (http://www. jach.hawaii.edu/UKIRT/MHCat/), where they are listed as MHO 1605 to 1610.

The two most prominent $\mathrm{H}_{2}$ jets, MHO 1607 and MHO 1608, are found in the north-western part of the cluster $\mathrm{Cr}$ 232. A comparison of the $\mathrm{H}_{2}$ image and the $\mathrm{Br} \gamma$ image is shown in Fig. 8. The fact that these features are bright in the $2.121 \mu \mathrm{m} \mathrm{H}_{2}$ line but not visible in the $\mathrm{Br} \gamma$ line confirms the nature of collision (i.e. jet-shock) induced emission.

MHO 1607 (J104428.2-593245) is composed of (at least) five discernable knots that extend over about 6 ", approximately in east-west direction. The morphology seems to suggest that the jet is moving towards the west. The HAWK-I images do not reveal an obvious candidate for the source of the flow. The second object, MHO 1608 (J104429.1-593242), is more compact and less bright. It seems possible that these two MHOs belong to a single flow system. In that case, the total linear extent is about $11^{\prime \prime}$ and corresponds to a physical length of (at least) $0.12 \mathrm{pc}$.
Figure 8 shows that the surface of the clouds surrounding $\mathrm{Cr} 232$ emits in the $\mathrm{H}_{2}$ line and also in the $\mathrm{Br} \gamma$ line, showing that this is fluorescent emission from the ionization front at the surface of the cloud. In addition to the presence of the deeply embedded YSOs in this cloud (see Preibisch et al. 2011c), the detection of these jets clearly shows that $\mathrm{Cr} 232$ is a prominent site of actively ongoing star formation in the $\mathrm{CNC}$.

Figure 9 shows the other four MHOs detected in the HAWK-I images. MHO 1605 (J104351.4-593911) is located south of $\operatorname{Tr} 14$. The jet is embedded in the south-eastern tip of an hour-glass shaped globule. Two patches of $\mathrm{H}_{2}$ emission are seen above and below the rim of the globule, and a faint point-like source is seen inside the globule and just between them. MHO 1606 (J104422.7-593354) is located south-west of Cr 232. It consists of a diffuse knot with a weak $2^{\prime \prime}$ extension to the north-east. MHO 1610 (J104608.4-594524) is located on the northern rim of a large pillar in the Tr 16 region. It consists of two diffuse blobs separated by $1.5^{\prime \prime}$.

MHO 1609 (J104510.1-600229) is located in the middle of a small elongated cloud in the western parts of the South Pillars region. This is the only case of a jet found in the central region of a globule. The projected length of the flow system is $\approx 10^{\prime \prime}$, corresponding to a physical length of (at least) $0.11 \mathrm{pc}$. We note that this cloud is the source of the five optical jet candidates HHc-4 to HHc- 8 described by Smith et al. (2010a), but the MHO seems to be unrelated to these optical $\mathrm{HH}$ objects. On the other hand, for none of the optical HH object candidates do we see any molecular hydrogen emission. The HAWK-I images show a faint diffuse point-like source that may be related to HHc-6, but since this object is similarly bright in the $\mathrm{H}_{2}$ and $\mathrm{Br} \gamma$ images, what we see is not $\mathrm{H}_{2}$ line emission but probably reflected light (perhaps the envelope of the jet-driving protostar). 


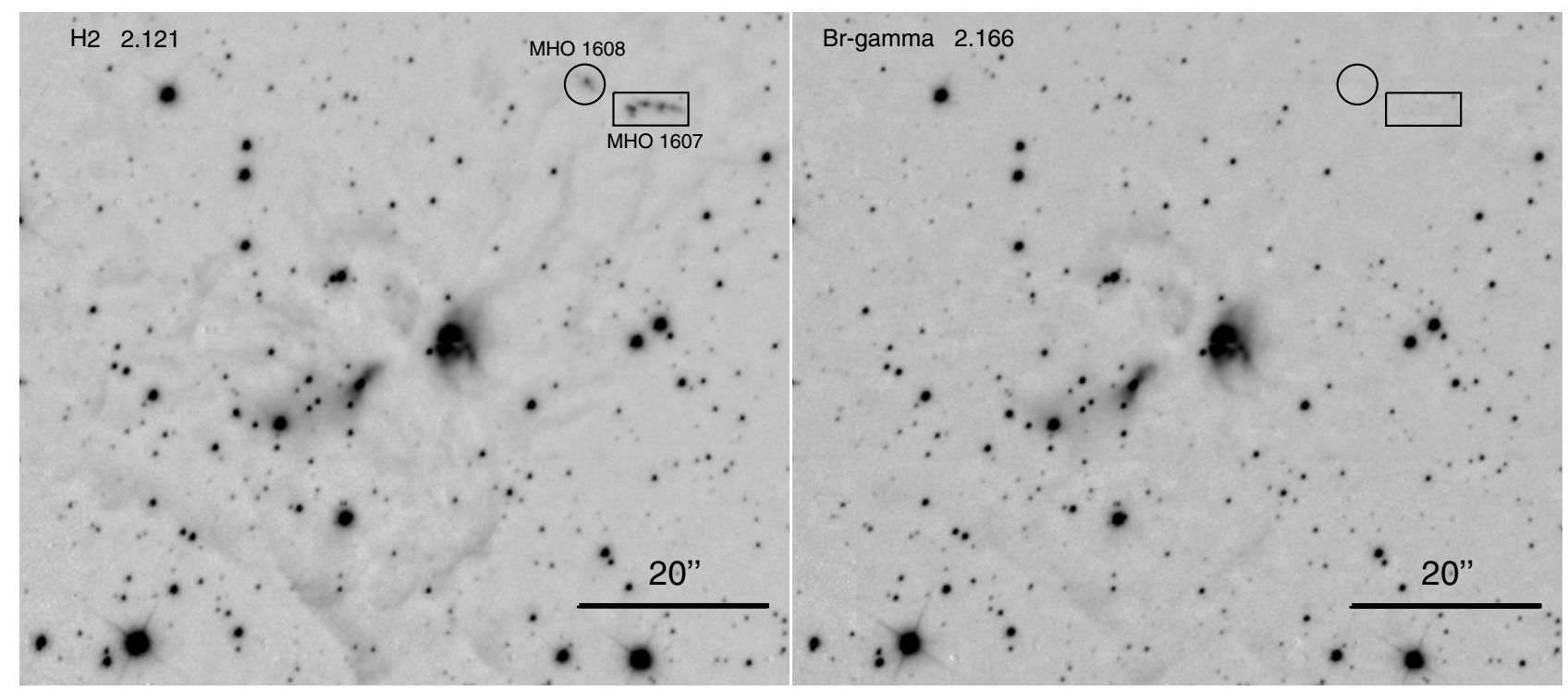

Fig. 8. Images of the $\mathrm{Cr} 232$ region obtained through the $2.121 \mu \mathrm{m} \mathrm{H}_{2}$ narrow-band filter (left $)$ and the $2.166 \mu \mathrm{m} \mathrm{Br} \gamma$ narrow-band filter (right). The location of the two molecular hydrogen jets found in this area, MHO 1607 (104428.2-593245) and MHO 1608 (104429.1-593242), are marked by the box and the circle in the upper right part of the images. The bright extended object just right of the center is the edge-on circumstellar disk object described in detail in Preibisch et al. (2011c).

\subsection{Search for $\mathrm{H}_{2}$ emission from the optical $\mathrm{HH}$ objects}

The HST H $\alpha$ imaging survey of Smith et al. (2010a) revealed $39 \mathrm{HH}$ jets and jet candidates from YSOs that are embedded in dense globules. 13 of these $\mathrm{HH}$ jets and 14 jet candidates are located in the area covered by our HAWK-I narrow-band images. Our detailed inspection of the surroundings of the optical jets in the HAWK-I images showed that several of the optical HH jets can also be seen in the NIR narrow-band images, but in all cases the apparent brightness in the $\mathrm{Br} \gamma$ line filter is similar to that in the $\mathrm{H}_{2}$ line filter. This implies that the emission we see in the $\mathrm{H}_{2}$ filter image is not shock-induced but either related to UV fluorescence or just reflected continuum light. This is most likely a consequence of the fact that the optical HH jets seen by HST are irradiated atomic jets that have left the globules and expand in the surrounding diffuse atomic interstellar medium. This implies that no molecular hydrogen should be present in the immediate surroundings of the $\mathrm{HH}$ jets, and thus no $\mathrm{H}_{2}$ line emission is expected.

However, one might expect to see $\mathrm{H}_{2}$ line emission from those parts of the jet flow that are still within the dense globules (i.e. the part of the path before the jets emerge into the surrounding diffuse atomic medium). Therefore we performed a detailed inspection of those parts of the globules from which the optical $\mathrm{HH}$ jets emerge. If the jet-driving protostars were located deeply inside these globules, one would expect to see $\mathrm{H}_{2}$ line emission within the globule that should be aligned with the optical HH emission outside the surface of the globule. However, in no case do we find detectable $\mathrm{H}_{2}$ line emission at such locations.

This null result is in agreement with the fact that none of the optical $\mathrm{HH}$ jets exhibited detectable excess $4.5 \mu \mathrm{m}$ emission (which is also related to ro-vibrational lines from molecular hydrogen) in the Spitzer imaged analyzed by Smith et al. (2010b). This has an interesting implication: it shows that the driving sources of these jets are located very close to the edge of the globules, and not in the center of the globules (because then we would expect to see MHOs). For a protostar located at the edge (or at the tip) of a globule, the jets will immediately move into the diffuse atomic medium, and no MHOs are thus expected.

\subsection{Implications on the star formation mechanism}

We find only a very small number of six MHOs, i.e. signposts of protostellar jets, in the molecular clouds in the CNC. The clear lack of $\mathrm{H}_{2}$ jet detections at the positions of the numerous optical $\mathrm{HH}$ jets suggests that the driving sources are located very close to the surface of the globules, and the jets therefore move in the atomic gas, not through the molecular gas in the globules. This implies that almost all jet-driving protostars (i.e. currently forming stars) are located very close to the irradiated edges of the clouds, and not in the central regions of the clouds.

This spatial configuration strongly supports the scenario that star formation is currently triggered by the advancing ionization front in the irradiated clouds (see Smith et al. 2010b). This scenario predicts that stars form very close to the irradiated edge of the clouds, where the radiative compression leads to collapse and triggers star formation. In the alternative scenario, if one would assume spontaneous star formation taking place at random locations within these clouds, most protostars should be located in the inner parts of the clouds and we would thus expect to see more cases of MHOs within the clouds, which is not the case.

\section{Summary and conclusions}

\subsection{Summary of the main results}

The analysis of the infrared properties of the young stars in the CNC provides important information about the stellar ages and masses, as well as the mass function and the total size of the stellar population. The ages estimated for the low-mass populations in the young clusters within the $\mathrm{CNC}$ are consistent with previous age determinations for the massive cluster members, suggesting that the high- and low-mass stars have the same age, i.e. have formed together at the same time.

The number of X-ray detected stars with CMD positions corresponding to stellar masses of $M_{*} \geq 1 M_{\odot}$ is consistent with an extrapolation of the field star IMF based on the number of high-mass $\left(M \geq 20 M_{\odot}\right)$ stars. This suggests that the IMF in the CNC is consistent with the field star IMF (down to at least 
T. Preibisch et al.: Deep wide-field near-infrared survey of the Carina Nebula

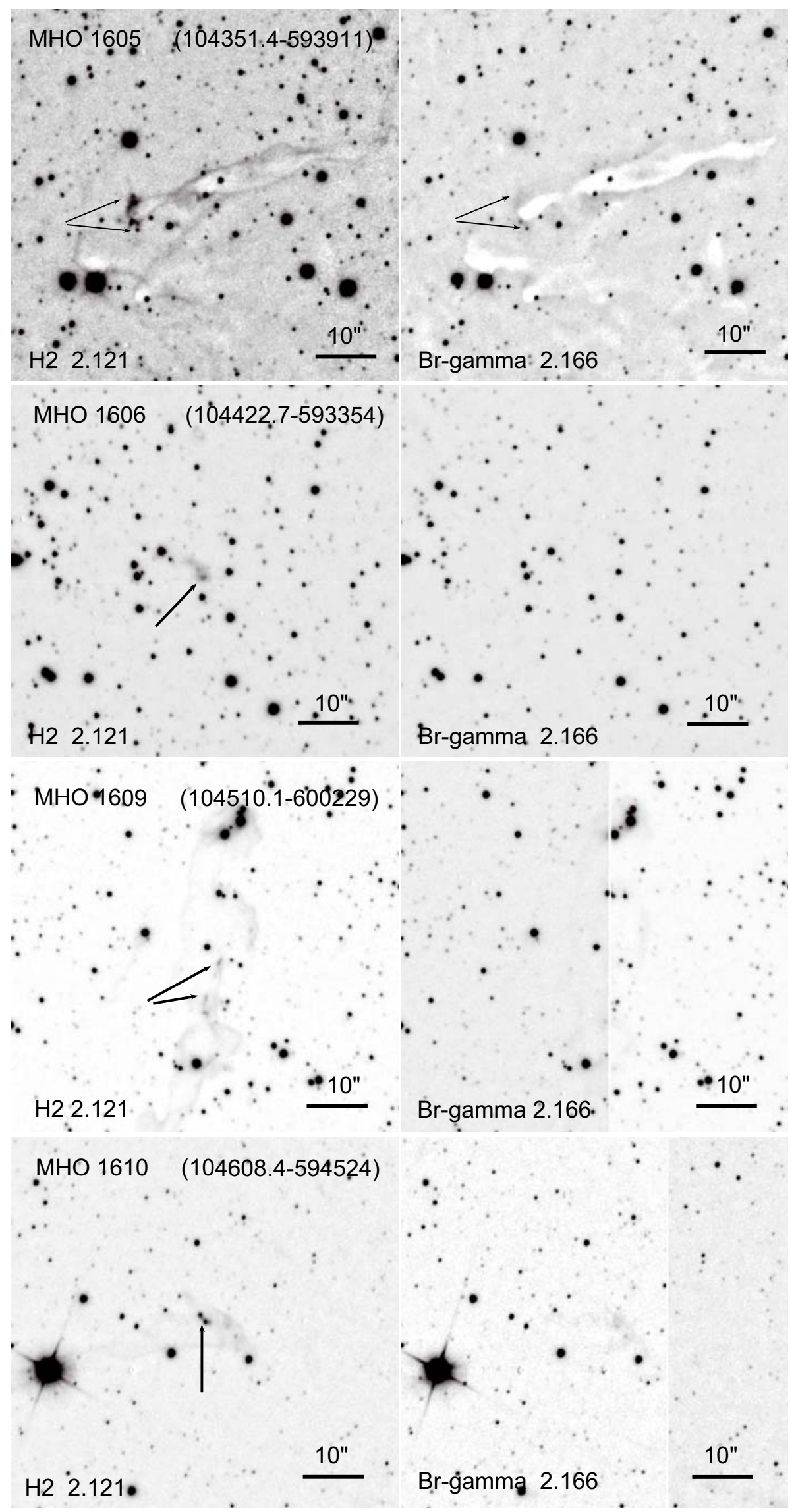

Fig. 9. Images of the jets MHO 1605, 1606, 1609 , and 1610 , obtained through the $2.121 \mu \mathrm{m}$ $\mathrm{H}_{2}$ narrow-band filter (left) and the $2.166 \mu \mathrm{m}$ $\mathrm{Br} \gamma$ narrow-band filter (right). The location of the molecular hydrogen jets are marked by the arrows. 
$\left.1 M_{\odot}\right)$. The extrapolation of the X-ray detected population down to $0.1 M_{\odot}$ suggests a total population of $\approx 43730$ stars with an integrated mass of about $28000 M_{\odot}$. We note that this extrapolation only considers the star numbers in the X-ray detected, lightly obscured population of young stars in the CNC. The analysis of deep Spitzer images of the CNC by Povich et al. (2011a) revealed a population of 1439 YSO candidates with strong mid-infrared excesses that is thought to be dominated by intermediate-mass $\left(2 \ldots 10 M_{\odot}\right)$ stars. Most $(72 \%)$ of these are not detected in the Chandra X-ray data, probably due to their strong obscuration. The extrapolations of Povich et al. (2011a) suggests a total population of $\sim 14300$ obscured objects, that have to be added to the $\mathrm{X}$-ray selected population. This raises the total population of the CNC to $\approx 58000$ stars, and the integrated stellar mass to $\approx 37000 M_{\odot}$. These numbers ${ }^{5}$ clearly show that the CNC constitutes one of the most massive galactic clusters/associations, probably topping the often quoted galactic "starburst templates" NGC $3603\left(M_{* \text {,tot }} \sim 15000 M_{\odot}\right.$, see Rochau et al. 2010) and the Arches cluster ( $M_{* \text {,tot }} \sim 20000 M_{\odot}$, see Espinoza et al. 2009), and being similar to Westerlund 1 $\left(M_{*, \text { tot }} \sim 49000 M_{\odot}\right.$, see Gennaro et al. 2011). The CNC can thus be regarded as the most nearby galactic cluster on the verge of extragalactic starburst clusters.

The presence of several deeply embedded young stellar objects and the detection of jets show that the cloud associated to the cluster $\mathrm{Cr} 232$ is a center of ongoing star formation activity.

The fact that we find only four new small clusters in the HAWK-I images confirm and extend previous results showing that about half of the total stellar population in the CNC is in a non-clustered, widely dispersed spatial mode. The presence of this large distributed stellar population suggests that the $\mathrm{CNC}$ should be considered as an unbound $\mathrm{OB}$ association rather than a cluster of clusters. Since most of the existing clusters are quite loose and will probably disperse within a few $10 \mathrm{Myr}$, the fraction of widely distributed, non-clustered stars will even increase with time.

The very small number of $\mathrm{H}_{2}$ jets we see in the clouds and the complete lack of molecular hydrogen emission related to the numerous known optical HH jets show that most of the very recently formed protostars must be located very close to the surface of the globules (not inside), suggesting a triggered star formation mechanism.

\subsection{Interesting aspects of the current star formation activity in the CNC}

The UV radiation and winds of the very hot and luminous early O-type and WR stars in the central area of the CNC affect the surrounding clouds strongly. While much of the cloud mass was (and still is) dissolved and streams away in the expanding super-bubble, the remaining molecular cloud material is now highly fragmented into numerous dense pillars. The total (dust + gas) mass of the dense clouds in the CNC is still quite large $\left(\sim 60000 M_{\odot}\right)$, but these clouds fill only a small fraction of the volume of the CNC (see Preibisch et al. 2011a). The radiative compression of these clouds currently leads to triggered star formation. The spatial distribution of the YSOs detected in the Spitzer survey (Smith et al. 2010b) suggests that the induced

\footnotetext{
5 As a historical note, it is interesting to compare this number to older estimates based on optical observations only: for example, Feinstein (1995) estimated the total stellar mass of the CNC to be $3596 M_{\odot}$. The progress in observations has lead to an increase by more than a factor of 10 within just about 15 years!
}

formation of stars happens predominantly near the most strongly irradiated tips of the clouds. This is consistent with recent results from numerical simulations of the evolution of irradiated clouds (e.g., Gritschneder et al. 2010). An implication of this triggered star formation mechanism is that the cloud material surrounding the newly formed protostars is simultaneously dissolved by the advancing ionization front. As their natal surrounding clouds and protostellar envelopes are dispersed very quickly, these newly formed stars are immediately exposed to the very harsh radiation field created by the luminous O-type stars, and therefore loose their circumstellar material very quickly. The transition from an embedded protostellar object to a revealed disk-less star thus happens faster than in star forming regions with lower levels of feedback. This can explain the very small fraction of young stars with NIR excesses as a tracer of circumstellar disks.

Several aspects of this process of triggered formation of a new stellar generation in the $\mathrm{CNC}$ appear particularly interesting and are worth being discussed in more detail. First, the triggering process has produced many sparse groups of young stars instead of a few rich clusters; it is thus a qualitatively different mode of star formation than the process that formed the earlier generation of stars in the much more populous clusters $\operatorname{Tr} 16$ (and $\operatorname{Tr} 14)$. The relatively small sizes of the newly formed clusterings of young stars suggests that the currently ongoing radiative triggering of star formation is a very local, small-scale process. This is probably related to the highly fragmented structure of the now existing dense clouds (as can be seen in the sub-mm map in Preibisch et al. 2011a). The compression by the ionization front drives theses rather small individual clouds at various locations in the nebula into collapse, each of which then gives birth to a small stellar group.

The second aspect is that the masses of the stars in the triggered new generation seem to be restricted to $\lesssim 20 M_{\odot}$, i.e. to much lower values than the very massive stars in the older, triggering generation $\left(M \gtrsim 100 M_{\odot}\right.$ for $\eta$ Car and the $\mathrm{O} 3$ and WR stars in Tr 16 and Tr 14). The infrared and X-ray surveys revealed several candidates for intermediate-mass and moderately high-mass YSOs, but no trace of any very massive $\left(M \geq 50 M_{\odot}\right)$ protostar has yet been found. This is again supported by the results of the sub-mm study of the structure of the dense clouds (Preibisch et al. 2011a), where it was found that nearly all dense clouds in the CNC have masses $\leq 1000 M_{\odot}$; according to the empirical relation between cloud mass and maximum stellar mass, these clouds are expected to yield maximum stellar masses of no more than $\lesssim 15 M_{\odot}$.

The third aspect concerns the size of the triggered new stellar population. The X-ray survey showed that the widely distributed population consists of a similar number $(\sim 5000 \mathrm{X}$-ray detected objects) of stars as the populations of the triggering populations in $\operatorname{Tr} 16+\operatorname{Tr} 14$. Considering the spatial incompleteness effect discussed in Sect. 3.7, the dispersed population is probably even somewhat larger than the cluster populations. Evidence for a second generation of star formation triggered by radiative and windfeedback from the massive stars in a first generation has been found in several other massive star forming regions, but in most cases the total size of the second stellar generation is considerably smaller than that of the first generation (see, e.g., Stanke et al. 2002; Reach et al. 2004; Linsky et al. 2007; Wang et al. 2010). The remarkably large size of the latest stellar generation in the CNC may be related to the particularly high level of massive star feedback in the $\mathrm{CNC}$, that is perhaps more effective in triggering star formation than the much lower feedback levels in regions with smaller populations of very massive stars in the older generation. 
The combination of the deep HAWK-I NIR images with the results of the X-ray survey has provided us with a very substantial amount of crucial new information about the stellar content, the star formation history, and the ongoing star formation process in the CNC. Future studies will combine these observational data with detailed numerical simulations of how molecular clouds evolve under the influence of strong massive star feedback (see Gritschneder et al. 2010). Such a comparison can provide new and detailed insights into fundamental processes such as the disruption of molecular clouds by massive stars, the origin of the observed complex pillar-like structures at the interfaces between molecular clouds and $\mathrm{H}$ II regions, the effect of stellar feedback on molecular cloud dynamics and turbulence, and how ionizing radiation and stellar winds trigger the formation of a new generation of stars.

Acknowledgements. We would like to thank the referee for a very competent review and several suggestions that helped to improve this paper. We thank the ESO staff (especially Markus Kissler-Patig and Monika Petr-Gotzens) for performing the HAWK-I observations in service mode. We gratefully acknowledge funding of this work by the German Deutsche Forschungsgemeinschaft, $D F G$ project number PR 569/9-1. Additional support came from funds from the Munich Cluster of Excellence: "Origin and Structure of the Universe". R.R.K. is supported by a Leverhulme research project grant (F/00 144/BJ).

\section{References}

Adams, F. C. 2010, ARA\&A, 48, 47

Ascenso, J., Alves, J., Vicente, S., \& Lago, M. T. V. T. 2007, A\&A, 476, 199 Bally, J., Sutherland, R. S., Devine, D., \& Johnstone, D. 1998, AJ, 116, 293 Baraffe I., Chabrier G., Allard F., \& Hauschildt P. H. 1998, A\&A, 337, 403 Baraffe, I., Chabrier, G., \& Gallardo, J. 2009, ApJ, 702, L27

Bastian, N., Covey, K. R., \& Meyer, M. R. 2010, ARA\&A, 48, 339

Baume, G., Vázquez, R. A., Carraro, G., \& Feinstein, A. 2003, A\&A, 402, 549

Bessel, M. S., \& Brett, J. M. 1988, PASP, 100, 1134

Blaauw, A. 1964, ARA\&A, 2, 213

Black, J. H., \& Dalgarno, A. 1976, ApJ, 203, 132

Bressert, E., Bastian, N., Gutermuth, R., et al. 2010, MNRAS, 409, L54

Briceño, C., Calvet, N., Hernández, J., et al. 2005, AJ, 129, 907

Briceño C., Preibisch, Th., Sherry, W., et al. 2007, in Protostars \& Planets V, ed. B. Reipurth, D. Jewitt, \& K. Keil (Tucson: University of Arizona Press), 345 Brooks, K. J., Garay, G., Nielbock, M., Smith, N., \& Cox, P. 2005, ApJ, 634, 436

Broos, P. S., Feigelson, E. D., Townsley, L. K., et al. 2007, ApJS, 169, 353

Broos, P. S., Townsley, L. K., Feigelson, E. D., et al. 2011a, ApJS (CCCP Special Issue), in press [arXiv: 1102.5121]

Broos, P. S., Getman, K. V., Povich, M. S., et al. 2011b, ApJS (CCCP Special Issue), in press [arXiv: 1102.5120$]$

Clarke, C. J. 2007, MNRAS, 376, 1350

Currie, T., Hernandez, J., Irwin, J., et al. 2010, ApJS, 186, 191

Davis, C. J., Scholz, P., Lucas, P., Smith, M. D., \& Adamson, A. 2008, MNRAS, 387,954

DeGioia-Eastwood, K., Throop, H., Walker, G., \& Cudworth, K. M. 2001, ApJ, 549,578

Dias, W. S., Alessi, B. S., Moitinho, A., \& Lépine, J. R. D. 2002, A\&A, 389, 871

Espinoza, P., Selman, F. J., \& Melnick, J. 2009, A\&A, 501, 563

Feigelson, E. D., Townsley, L., Güdel, M., \& Stassun, K. 2007, Protostars \& Planets V, ed. B. Reipurth, D. Jewitt, \& K. Keil (Univ. Arizona Press), 313

Feigelson, E. D., Getman, K. V., Townsley, L. K., et al. 2011, ApJS (CCCP Special Issue), in press [arXiv: 1103.0802]

Feinstein, A. 1995, Rev. Mex. Astron. Astrofis. Conf. Ser., 2, 57

Fitzgerald, M. P., \& Mehta, S. 1987, MNRAS, 228, 545

Forbrich, J., \& Preibisch, T. 2007, A\&A, 475, 959

Freyer, T., Hensler, G., \& Yorke, H. W. 2003, ApJ, 594, 888

Garcia Lopez, R., Nisini, B., Eislöffel, J., et al. 2010, A\&A, 511, A5

Gennaro, M., Brandner, W., Stolte, A., \& Henning, T. 2011, MNRAS, 162

Getman, K. V., Flaccomio, E., Broos, P. S., et al. 2005, ApJS, 160, 319

Gritschneder, M., Burkert, A., Naab, T., \& Walch, S. 2010, ApJ, 723, 971

Guarcello, M. G., Prisinzano, L., Micela, G., et al. 2007, A\&A, 462, 245

Gutermuth, R. A., Megeath, S. T., Myers, P. C., et al. 2009, ApJS, 184, 18
Haisch, K. E., Jr., Lada, E. A., \& Lada, C. J. 2000, AJ, 120, 1396

Haisch, K. E., Lada, E. A., Pina, R. K., Telesco, C. M., \& Lada, C. J. 2001, AJ, 121,1512

Hartmann, L. 2001, AJ, 121, 1030

Hartmann, L. 2003, ApJ, 585, 398

Hillenbrand, L. A., Bauermeister, A., \& White, R. J., Proc. Cool Stars 15 [arXiv:astro-ph/0703642]

Hodgkin, S. T., Irwin, M. J., Hewett, P. C., \& Warren, S. J. 2009, MNRAS, 394, 675

Irwin, M. J. 1985, MNRAS, 214, 575

Irwin, M. J., Lewis, J., Hodgkin, S., et al. 2004, Proc. SPIE, 5493, 411

Kissler-Patig, M., Pirard, J.-F., Casali, M., et al. 2008, A\&A, 491, 941

Kroupa, P. 2002, Science, 295, 82

Lane, K. P., Almaini, O., Foucaud, S., et al. 2007, MNRAS, 379, L25

Lejeune T., \& Schaerer D. 2001, A\&A, 366, 538

Linsky, J. L., Gagné, M., Mytyk, A., McCaughrean, M., \& Andersen, M. 2007, ApJ, 654, 347

Liu, Q., de Grijs, R., Deng, L. C., et al. 2009, MNRAS, 396, 1665

Lyo, A.-R., Lawson, W. A., Mamajek, E. E., et al. 2003, MNRAS, 338, 616

Martins, F., Schaerer, D., \& Hillier, D. J. 2005, A\&A, 436, 1049

Massey, P., DeGioia-Eastwood, K., \& Waterhouse, E. 2001, AJ, 121, 1050

McCaughrean, M. J., Rayner, J. T., \& Zinnecker, H. 1994, ApJ, 436, L189

Mentuch, E., Abraham, R. G., Glazebrook, K., et al. 2009, ApJ, 706, 1020

Miller G. E., \& Scalo J. M. 1978, PASP, 90, 506

Mottram, J. C., Hoare, M. G., Lumsden, S. L., et al. 2007, A\&A, 476, 1019

Nisini, B., Caratti o Garatti, A., Giannini, T., \& Lorenzetti, D. 2002, A\&A, 393 1035

Oliveira, I., Merin, B., Pontoppidan, K. M., et al. 2009, ApJ, 691, 672

Povich, M. S., Smith, N., Majewski, S. R., et al. 2011a, ApJS (CCCP Special Issue), in press [arXiv: 1103.2060 ]

Povich, M. S., Townsley, L. K., Broos, P. S., et al. 2011b, ApJS (CCCP Special Issue), in press [arXiv: 1102 .5366]

Preibisch, Th., \& Zinnecker, H. 1999, AJ, 121, 1040

Preibisch, Th., \& Zinnecker H. 2002, AJ, 123, 1613

Preibisch, T., \& Zinnecker, H. 2007, IAU Symp., 237, 270

Preibisch, Th., Zinnecker, H., \& Herbig, G. H. 1996, A\&A, 310, 456

Preibisch, Th., Brown, A. G. A., Bridges, T., Guenther, E., \& Zinnecker, H. 2002, AJ, 124, 404

Preibisch, Th., Kim, Y.-C., Favata, F., et al. 2005a, ApJS, 160, 401

Preibisch, Th., Schuller, F., Ohlendorf, H., et al. 2011a, A\&A, 525, A92

Preibisch, Th., Hodgkin, S., Irwin, M., et al. 2011b, ApJS (CCCP Special Issue), in press [arXiv: 1103.2052]

Preibisch, Th., Ratzka, T., Gehring, T., et al. 2011c, A\&A, in press [arXiv: 1104.3706]

Reach, W. T., Rho, J., Young, E., et al. 2004, ApJS, 154, 385

Rebull, L. M., Padgett, D. L., McCabe, C.-E., et al. 2010, ApJS, 186, 259

Rochau, B., Brandner, W., Stolte, A., et al. 2010, ApJ, 716, L90

Sabbi, E., Sirianni, M., Nota, A., et al. 2008, AJ, 135, 173

Sana, H., Momany, Y., Gieles, M., et al. 2010, A\&A, 515, A26

Sanchawala, K., Chen, W.-P., Ojha, D., et al. 2007b, ApJ, 667, 963

Selman, F. J., \& Melnick, J. 2005, A\&A, 443, 851

Siess, L., Dufour, E., \& Forestini, M. 2000, A\&A, 358, 593

Slesnick, C. L., Hillenbrand, L. A., \& Carpenter, J. M. 2008, ApJ, 688, 377

Smith, N. 2006, MNRAS, 367, 763

Smith, M. D., O'Connell, B., \& Davis, C. J. 2007, A\&A, 466, 565

Smith, N., Stassun, K. G., \& Bally, J. 2005, AJ, 129, 888

Smith, N., \& Brooks, K. J. 2008, in Handbook of Low Mass Star Forming Regions, Vol. II: The Southern Sky, ed. B. Reipurth, ASP Monograph Publications, 5, 138

Smith, N., Bally, J., \& Walborn, N. R. 2010a, MNRAS, 405, 1153

Smith, N., Powich, M. S., Whitney, B. A., et al. 2010b, MNRAS, 789

Stanke, T., Smith, M. D., Gredel, R., \& Szokoly, G. 2002, A\&A, 393, 251

Tapia, M., Roth, M., Vazques, R. A., \& Feinstein, A. 2003, MNRAS, 339, 444

Throop, H. B., \& Bally, J. 2005, ApJ, 623, L149

Townsley, L., Broos, P. S., Corcoran, M. F., et al. 2011, ApJS (CCCP Special Issue), in press [arXiv: 1102 .4779]

Wang, J., Feigelson, E. D., Townsley, L. K., et al. 2010, ApJ, 716, 474

Wang, J., Feigelson, E. D., Townsley, L. K., et al. 2011, ApJS (CCCP Special Issue), in press [arXiv: 1103.0785]

Whitworth, A. P., \& Zinnecker, H. 2004, A\&A, 427, 299

Wolk, S. J., Harnden, F. R., Jr., Flaccomio, E., et al. 2005, ApJS, 160, 423

Wolk, S. J., Broos, P. S., Getman, K. V., et al. 2011, ApJS (CCCP Special Issue), in press [arXiv: 1103.1126]

Yonekura, Y., Asayama, S., Kimura, K., et al. 2005, ApJ 634, 476 